\title{
Structure and stability of the compressible Stuart vortex
}

\author{
By G. O'REILLY AND D. I. PULLIN \\ Graduate Aeronautical Laboratories, 105-50, California Institute of Technology, \\ Pasadena, CA 91125, USA \\ goreilly@galcit.caltech.edu
}

(Received 30 December 2002 and in revised form 20 May 2003)

The structure and two- and three-dimensional stability properties of a linear array of compressible Stuart vortices (CSV; Stuart 1967; Meiron et al. 2000) are investigated both analytically and numerically. The CSV is a family of steady, homentropic, two-dimensional solutions to the compressible Euler equations, parameterized by the free-stream Mach number $M_{\infty}$, and the mass flux $\epsilon$ inside a single vortex core. Known solutions have $0<M_{\infty}<1$. To investigate the normal-mode stability of the generally spatially non-uniform CSV solutions, the linear partial-differential equations describing the time evolution of small perturbations to the CSV base state are solved numerically using a normal-mode analysis in conjunction with a spectral method. The effect of increasing $M_{\infty}$ on the two main classes of instabilities found by Pierrehumbert \& Widnall (1982) for the incompressible limit $M_{\infty} \rightarrow 0$ is studied. It is found that both two- and three-dimensional subharmonic instabilities cease to promote pairing events even at moderate $M_{\infty}$. The fundamental mode becomes dominant at higher Mach numbers, although it ceases to peak strongly at a single spanwise wavenumber. We also find, over the range of $\epsilon$ investigated, a new instability corresponding to an instability on a parallel shear layer. The significance of these instabilities to experimental observations of growth in the compressible mixing layer is discussed. In an Appendix, we study the CSV equations when $\epsilon$ is small and $M_{\infty}$ is finite using a perturbation expansion in powers of $\epsilon$. An eigenvalue determining the structure of the perturbed vorticity and density fields is obtained from a singular Sturm-Liouville problem for the stream-function perturbation at $O(\epsilon)$. The resulting small-amplitude steady CSV solutions are shown to represent a bifurcation from the neutral point in the stability of a parallel shear layer with a tanh-velocity profile in a compressible inviscid perfect gas at uniform temperature.

\section{Introduction}

The incompressible turbulent mixing layer has been the subject of many experimental investigations. Winant \& Browand (1974) studied an incompressible mixing layer at moderate Reynolds number, and found large spanwise-organized vortical structures. Brown \& Roshko (1974) demonstrated the existence of these spanwise structures at large values of the Reynolds numbers. It has been shown that these large eddies are principally responsible for boosting the growth rates of incompressible shear layers and fluid entrainment into the mixing region, via pairing and amalgamations: 
Winant \& Browand (1974), Brown \& Roshko (1974), Browand \& Latigo (1979), Bernal \& Roshko (1986) and Moore \& Saffman (1972).

With the advent of high-speed propulsion systems, experiments involving compressible mixing layers gained importance. Birch \& Eggers (1972) compiled a survey of free turbulent shear layer data and showed that as the Mach number increased, the turbulent growth rate decreased. This was initially thought to be due to the different free-stream densities used to increase the free-stream Mach number. Brown \& Roshko (1974) showed that the large decrease in growth rates was produced by increasing compressibility, not by density effects. Bogdanoff (1983) and Papamoschou \& Roshko (1988) used the concept of convective Mach number, $M_{c}$, to investigate this reduction in growth rates, finding good collapse in the growthrate data. More recently Slessor, Zhuang \& Dimotakis (2000) suggest a new scaling parameter suitable for flows with free-stream density and sound-speed ratios far from unity. Gas compressibility also produces changes in the large-scale vortex structures in mixing layers. Experimental evidence for the form of these structures is not definitive: Goebel \& Dutton (1991), Samimy \& Elliott (1990), Hall, Dimotakis \& Rosemann (1993), Clemens \& Mungal (1995) and Papamoschou \& Bunyajitradulya (1996). It is clear from these experiments that the large-scale structures become more three-dimensional as $M_{c}$ increases.

Much numerical and analytical work has been done using parallel models for the compressible shear layer: Lees \& Lin (1946), Lin (1953), Lessen, Fox \& Zien (1965, 1966), Blumen (1970), Blumen, Drazin \& Billings (1975), Sandham \& Reynolds (1991, 1990), Zhuang, Dimotakis \& Kubota (1990a), Zhuang, Kubota \& Dimotakis (1990b) and Zhuang \& Dimotakis (1995). These works show a clear decrease in linear growth rates as $M_{c}$ increases, and further, that three-dimensional instabilities are more vigorously amplified at higher Mach numbers. Non-parallel base flows, including the incompressible Stuart vortex and Kelvin-Helmholtz billows, have been used to determine the two- and three-dimensional stability properties of incompressible free shear layers: Pierrehumbert \& Widnall (1982), Klaassen \& Peltier (1985, 1989, 1991). These works illustrate the effect and importance of secondary instabilities, related to the organized vortical structures present in incompressible mixing layers, in the onset of three-dimensional turbulent flow. In the present work we utilize a non-parallel flow model for the compressible shear layer in an effort to study the role of compressibility in suppressing some of these secondary instabilities. This model is the compressible Stuart vortex (CSV) proposed by Meiron, Moore \& Pullin (2000) as a continuation to finite Mach number of the Stuart (1967) vortex.

The CSV is briefly reviewed in $\S 2$. Our approach to the numerical analysis of the instabilities of compressible shear flows is outlined in $\S 3$, and the numerical method is described. Parallel base flows are discussed in $\S 4$ and instabilities of the non-uniform steady flow represented by the CSV are described in $\S 5$, where comparisons are made with Pierrehumbert \& Widnall (1982) and Klaassen \& Peltier (1989) in the limit of very small Mach number. The effect of subsonic free-stream Mach number on the linear instabilities which seed pairing interactions, and which generate streamwise streaks, is examined. Increasing the Mach number will be seen to inhibit the effectiveness of the pairing instability to promote pairing events. At larger values of $M_{c}$, there does not appear to be one dominantly amplified spanwise wavelength for either of the linear instabilities. This may be a possible explanation for the disorganization of the large-scale structures as the Mach number increases. An analysis of the structure of the CSV, when the mass flux in the closed cat's-eye regions is small, is presented in the Appendix. 


\section{Compressible Stuart vortex (CSV)}

\subsection{Euler equations}

To facilitate the analysis of the CSV, we briefly review the formulation of Meiron et al. (2000, henceforth referred to as MMP), who considered the steady compressible Euler equations, together with the equation of state for a calorically perfect gas, for a shear flow in two-dimensional Cartesian coordinates $(x, y)$, with $x$ the streamwise and $y$ the transverse coordinate. The fluid velocity, vorticity, density, and pressure are denoted by $\boldsymbol{u}, \boldsymbol{\omega}, \rho$ and $p$ respectively, and * indicates a dimensional quantity. The subscript $\infty$ is used to refer to uniform reference quantities as $y \rightarrow \pm \infty$, where the flow consists of opposed uniform streams, each with speed $U_{\infty}^{*}$. In the following, unsubscripted fluid quantities are made non-dimensional using their reference values at infinity, and entropy is scaled with $c_{v}^{*}$. The free-stream Mach number is $M_{\infty}=U_{\infty}^{*} / a_{\infty}^{*}$.

MMP constructed a compressible continuation of the incompressible Stuart vortex for $M_{\infty}>0$. A stream function, $\psi(x, y)$-vorticity formulation of the steady compressible Euler equations was used where the velocity components are given by

$$
\rho u=\frac{\partial \psi}{\partial y}, \quad \rho v=-\frac{\partial \psi}{\partial x} .
$$

MMP then assumed homentropic flow and that the total enthalpy depends on $\psi(x, y)$ alone, $h \equiv h(\psi)$. A closed set of equations was obtained for the choice, $\mathrm{d} h / \mathrm{d} \psi=\mathrm{e}^{-2 \mu \psi}$, where $\mu$ is a parameter to be discussed subsequently. The momentum and energy equations may then be written as

$$
\begin{aligned}
\nabla^{2} \psi-\frac{1}{\rho}(\nabla \psi \cdot \nabla \rho) & =\rho^{2} \mathrm{e}^{-2 \mu \psi}, \\
\frac{M_{\infty}^{2}}{2}(\nabla \psi)^{2}+\frac{\rho^{2}\left(\rho^{\gamma-1}-1\right)}{\gamma-1} & =\frac{M_{\infty}^{2} \rho^{2}}{2}\left(1-\frac{1}{\mu} \mathrm{e}^{-2 \mu \psi}\right) .
\end{aligned}
$$

On the semi-infinite rectangle, $\{R: x \in[0, \pi], y \in[0, \infty]\}$, the boundary conditions are

$$
\begin{gathered}
\frac{\partial \psi}{\partial y}=0 \quad \text { on } \quad(y=0,0 \leqslant x \leqslant \pi), \quad \frac{\partial \psi}{\partial x}=0 \quad \text { on } \quad(x=0,0 \leqslant y \leqslant \infty), \\
\psi \sim y+d \quad \text { as } \quad(y \rightarrow \infty, 0 \leqslant x \leqslant \pi), \quad \rho \rightarrow 1 \quad \text { as } \quad(y \rightarrow \infty, 0 \leqslant x \leqslant \pi),
\end{gathered}
$$

which require symmetry about $y=0$. MMP show that two further conditions are needed to characterize solutions to (2.2)-(2.3). The first is to specify

$$
\epsilon=\psi(0,0)-\psi(\pi, 0)
$$

where $\epsilon, 0 \leqslant \epsilon<\infty$, is the mass flux inside the vortex core. The second is a constraint on the total dimensionless circulation. The unknowns are $\psi\left(x, y ; M_{\infty}, \epsilon\right), \rho\left(x, y ; M_{\infty}, \epsilon\right)$, $\mu\left(M_{\infty}, \epsilon\right)$, and $d\left(M_{\infty}, \epsilon\right)$.

\subsection{Incompressible Stuart vortex}

When $M_{\infty} \rightarrow 0$, with $\epsilon$ fixed, the solution to (2.2)-(2.3) is $\rho(x, y)=1, \mu=1$ and

$$
\psi=\ln \left(\kappa \cosh (y)+\sqrt{\kappa^{2}-1} \cos (x)\right), \quad \omega=-\left(\kappa \cosh (y)+\sqrt{\kappa^{2}-1} \cos (x)\right)^{-2} .
$$

The mass flux may be written as $\epsilon=2 \ln \left(\kappa+\sqrt{\kappa^{2}-1}\right)$, from which it is easily verified that $\kappa=\cosh (\epsilon / 2)$. This is the Stuart (1967) vortex. The parameter $\kappa \in(1, \infty)$ parameterizes the family of solutions. When $\kappa=1, \epsilon \rightarrow 0$, a parallel flow is obtained, $u=\tanh (y)$. When $\kappa \rightarrow \infty, \psi$ describes the potential flow produced by an infinite 
array of point vortices with spacing $2 \pi$. Intermediate $\kappa$ gives a smooth, periodic distribution of vorticity, where $\psi$ is even about the lines $x= \pm n \pi$ with $n$ integer. The steady streamline pattern is a periodic array of cat's eyes, with stagnation points on the symmetry line at $y=0$. The displacement thickness is $d=\ln (\kappa / 2)$.

\subsection{Homentropic continuation}

MMP found a continuation of the Stuart vortex to homentropic compressible flow by obtaining a family of solutions to (2.2)-(2.3) with two parameters, $M_{\infty}$, and $\epsilon$. For well-posedness, they found it necessary to treat $\mu\left(M_{\infty}, \epsilon\right)$ as an eigenvalue, its value being determined by solving the nonlinear governing equations. For a given $\epsilon$, their numerical solutions indicated a small range of subsonic $M_{\infty}$ for which locally supersonic smooth flow fields existed, while above some maximum, but subsonic, value of $M_{\infty}$ the solution branch was found to terminate. The termination is thought to be due to the onset of shocklets in the supersonic region, which would invalidate the governing equations. No two-dimensional solutions were found to exist for $M_{\infty} \geqslant 1$. For $M_{\infty} \ll 1$, at finite $\epsilon$, a Rayleigh-Janzen expansion showed that $\mu$ is determined from a solvability condition on the linearized equations, giving

$$
\mu_{0}\left(M_{\infty}\right)=1+\frac{1}{2} M_{\infty}^{2}+O\left(M_{\infty}^{4}\right) .
$$

To $O\left(M_{\infty}^{2}\right), \mu_{0}$ is independent of $\epsilon$. It follows that the limiting solution for the homentropic CSV when $\epsilon \rightarrow 0$ at finite $M_{\infty}$ is not given by its incompressible counterpart. This small-mass-flux limit was not resolved by MMP, and is analysed here in the Appendix, where it is also shown that this solution is intimately connected with the neutral stability point in the stability of parallel compressible flows.

The CSV has several limitations as a model of the nonlinear waves which are physically realizable in a compressible parallel flow. Both experiments and numerical simulations indicate that as $M_{c}$ is increased beyond 0.6 , the large-scale structures in the mixing layer become three-dimensional, a property which is not captured by the CSV. Furthermore, DNS of compressible vortices show entropy variations in the core, whereas the CSV is homentropic. Also, in a physical shear layer, the vorticity is compressed into thin regions, known as braids, between the vortex centres. The present two-dimensional CSV shows no such structures at the hyperbolic stagnation points. Nevertheless, the CSV still provides a useful model for examining the effect of compressibility on the interaction between neighbouring vortices in the compressible mixing layer environment.

\section{Linearized stability of the compressible Euler equations}

\subsection{Stability of non-uniform steady flows}

We now study the linearized stability of the CSV. The time evolution of small perturbations to solutions of (2.2)-(2.3), with finite $\epsilon$ and $M_{\infty}$, is considered. For the investigation of stability, the $\psi-\rho$ formulation is not appropriate and we utilize a primitive variable formulation in which, for given $M_{\infty}$ and $\epsilon$, the $2 \pi$-streamwise-periodic CSV base state is denoted by $(\bar{\rho}(x, y), \bar{u}(x, y), \bar{v}(x, y), \bar{s}(x, y), \bar{T}(x, y))$, and variables are non-dimensionalized as for the CSV. Small perturbations to the base state are denoted

$$
\begin{aligned}
\chi & \equiv\left[\rho^{\prime}, \boldsymbol{u}^{\prime}, s^{\prime}\right] \\
& \equiv\left[\rho^{\prime}(x, y, z, t), u^{\prime}(x, y, z, t), v^{\prime}(x, y, z, t), w^{\prime}(x, y, z, t), s^{\prime}(x, y, z, t)\right],
\end{aligned}
$$


where $z$ is the spanwise direction and $\boldsymbol{u}^{\prime}$ denotes the three velocity components. The perturbations, assumed to be isentropic, have a modal representation of the form

$$
\left[\rho^{\prime}, \boldsymbol{u}^{\prime}, s^{\prime}\right](x, y, z, t)=\mathrm{e}^{\mathrm{i} \alpha x} \mathrm{e}^{\mathrm{i} \beta z} \mathrm{e}^{-\sigma t}[\hat{\rho}, \hat{\boldsymbol{u}}, \hat{\boldsymbol{s}}](x, y) .
$$

For parallel base flows, the $x$ dependence of hatted quantities is dropped. For nonparallel base flows, the hatted quantities are taken to be periodic in $x$, with the same period as the base flow. The $y$ boundary conditions to be enforced are that as $y \rightarrow \pm \infty$ the hatted quantities decay to zero. No constraint will be placed on the parameters $\alpha$ and $\beta$, save that they be real. For non-parallel periodic base flows, $0 \leqslant \alpha<1$. The parameter $\beta$ is the wavenumber of disturbances in the spanwise direction. For parallel base flows, it may be coupled with $\alpha$ to define the angle of a particular disturbance $\theta$ as $\tan (\theta)=\beta / \alpha$. This does not have meaning for non-parallel base flows. No claim is made that perturbations (3.2) are complete or that perturbations do not exist which have an algebraic dependence on time.

The five linearized equations to be considered are the continuity, three momentum and entropy equations. Assuming (3.2) leads to an eigenvalue problem, with eigenvalue $\sigma=\sigma_{r}+\mathrm{i} \sigma_{i}$, the real part of which represents exponential growth/decay:

$$
\begin{gathered}
\left(\mathrm{L}_{1}+\nabla \cdot \bar{u}\right) \hat{\rho}+(\mathrm{i} \alpha \bar{\rho}+[\bar{\rho}, x]) \hat{u}+[\bar{\rho}, y] \hat{v}+\mathrm{i} \beta \bar{\rho} \hat{w}=\sigma \hat{\rho}, \\
\frac{1}{\bar{\rho}}\left(\mathrm{i} \alpha g+[g, x]+\mathrm{L}_{2} \bar{u}\right) \hat{\rho}+\left(\mathrm{L}_{1}+\frac{\partial \bar{u}}{\partial x}\right) \hat{u}+\left(\frac{\partial \bar{u}}{\partial y}\right) \hat{v}+\frac{1}{\bar{\rho}}(\mathrm{i} \alpha h+[h, x]) \hat{s}=\sigma \hat{u}, \\
\frac{1}{\bar{\rho}}\left([g, y]+\mathrm{L}_{2} \bar{v}\right) \hat{\rho}+\left(\frac{\partial \bar{v}}{\partial x}\right) \hat{u}+\left(\mathrm{L}_{1}+\frac{\partial \bar{v}}{\partial y}\right) \hat{v}+\frac{1}{\bar{\rho}}[h, y] \hat{s}=\sigma \hat{v}, \\
\frac{1}{\bar{\rho}} \mathrm{i} \beta g \hat{\rho}+\mathrm{L}_{1} \hat{w}+\frac{1}{\bar{\rho}} \mathrm{i} \beta h \hat{s}=\sigma \hat{w}, \\
\left(\frac{\partial \bar{s}}{\partial x}\right) \hat{u}+\left(\frac{\partial \bar{s}}{\partial y}\right) \hat{v}+\mathrm{L}_{1} \hat{s}=\sigma \hat{s} .
\end{gathered}
$$

The operators $\mathrm{L}_{1}, \mathrm{~L}_{2}$, and $[\cdot, \cdot]$, and the functions $g$ and $h$ may be defined as

$$
\begin{gathered}
\mathrm{L}_{1}=\mathrm{i} \alpha \bar{u}+\overline{\boldsymbol{u}} \cdot \nabla, \mathrm{L}_{2}=\bar{u} \frac{\partial}{\partial x}+\bar{v} \frac{\partial}{\partial y}, \quad[f, x]=\frac{\partial f}{\partial x}+f \frac{\partial}{\partial x}, \\
g(x, y)=\frac{1}{\gamma M_{\infty}^{2}}\left(1+(\gamma-1) \mathrm{e}^{\bar{s}(x, y)-\bar{s}_{\infty}}\right) \bar{\rho}^{\gamma-1}(x, y), \\
h(x, y)=\frac{1}{\gamma M_{\infty}^{2}} \mathrm{e}^{\bar{s}(x, y)-\bar{s}_{\infty}} \bar{\rho}^{\gamma-1}(x, y) .
\end{gathered}
$$

As $M_{\infty} \rightarrow 0$, the linearized equations approach a singular limit. For homentropic base flows the linearized entropy equation decouples from the remaining equations, implying

$$
\frac{\mathrm{d} s^{\prime}}{\mathrm{d} t}=0, \quad s^{\prime}(t) \equiv s(t, x(t), y(t)) \quad \text { where } \quad \dot{x}(t)=\bar{u}(x, y), \dot{y}(t)=\bar{v}(x, y) .
$$

Thus, a normal-mode assumption combined with the linear approximation imply, without loss of generality, that perturbations to a homentropic flow may be assumed to be homentropic.

Using similar arguments to those used by Pierrehumbert \& Widnall (1982, henceforth referred to as PW), some useful symmetry properties of equations (3.3)-(3.7) may be derived. Putting $\beta \rightarrow-\beta$, and reversing the sign of $\hat{w}$, an eigenfunction belonging to $\sigma$, for wavenumber $\beta$, may be turned into an eigenfunction belonging 
to the same eigenvalue, but now for wavenumber $-\beta$. Thus, it is only necessary to consider $\beta>0$. A similar argument gives $\alpha>0$. Finally, as a consequence of the time reversibility of the Euler equations, every exponentially damped mode has a corresponding exponentially growing mode. This means that the only stable modes are the neutral modes, $\sigma_{r}=0$.

\subsection{Numerical method}

To find the spectrum of equations (3.3)-(3.7), they must be approximated by a finite-order matrix, whose eigenvalues can be found using conventional methods. This is done using a spectral collocation technique. The perturbations, $(\hat{\rho}, \hat{\boldsymbol{u}}, \hat{s})$, are spectrally represented with basis functions that satisfy the boundary conditions. Spectral differentiation and integration is used to compute the individual components of the discretized matrix. The basis functions are not orthogonal; therefore, care must be taken to convert the resulting discretized system to standard eigenvalue form.

The techniques described, for non-parallel periodic base flows, are adapted from Boyd (1978a, b 1982) and Cain, Ferziger \& Reynolds (1984). The discretized perturbations are written as

$$
(\hat{\rho}, \hat{\boldsymbol{u}}, \hat{s})(x, y)=\sum_{m=-N_{x} / 2+1}^{N_{x} / 2} \sum_{n=0}^{N_{y}-3}\left(a_{m n}, \boldsymbol{b}_{m n}, c_{n m}\right) \mathrm{e}^{\mathrm{i} m x} \Phi_{n}(y),
$$

where $\Phi_{n}(y)$ are basis functions, which decay as $y \rightarrow \pm \infty$. The interval $(-1,1)$ is stretched onto $(-\infty, \infty)$ using the algebraic stretching, $Y=y / \sqrt{\eta^{2}+y^{2}}$, where $\eta$ is the stretching parameter. The functions $\Phi_{n}(y)$ are combinations of Chebyschev polynomials satisfying the boundary conditions. Letting $\phi_{n}(y(Y))=T_{n}(Y)$,

$$
\Phi_{n}(y)= \begin{cases}\phi_{0}(y)-\phi_{n+2}(y), & n \text { even } \\ \phi_{1}(y)-\phi_{n+2}(y), & n \text { odd }\end{cases}
$$

The number of collocation points in $y$ is $N_{y}$, chosen to be the zeros of the Chebyschev polynomial of order $N_{y}$, with $N_{x}$ points in $x$.

\subsection{Discrete matrix}

Discrete operators will be denoted with boldface sanserif symbols, and the discrete state vector denoted by $\boldsymbol{c}$. Thus equations (3.3)-(3.7) may be cast in the following non-standard eigenvalue form, where the growth rates $\sigma$ appear as the eigenvalues:

$$
\boldsymbol{A c}=\sigma \boldsymbol{B} \boldsymbol{c} .
$$

The matrix $\boldsymbol{A}$ is a block matrix, with $N_{e} \times N_{e}$ blocks, each representing a single term, multiplying any of $(\hat{\rho}, \hat{\boldsymbol{u}}, \hat{s})$, from the left-hand side of the system of equations (3.3)(3.7). Each block is dense and of size $N_{b} \times N_{b}$, where $N_{b}=N_{x}\left(N_{y}-2\right)$. Thus $\boldsymbol{A}$ is a $N \times N$ dense matrix, where $N=N_{e} N_{b}$. Storage requirements for $\boldsymbol{A}$ limited the maximum values of $N_{x}$ and $N_{y}$ which could be used.

Let $q(x, y)$ be a general function of the base flow, and of the parameters $\left(\alpha, \beta, M_{\infty}\right)$, defined from the left-hand side of equations (3.3)-(3.7). Then, the type of integral which must be considered in computing a general element from any of the blocks of $\boldsymbol{A}$ is

$$
I_{A}(k, s, m, n)=\int_{-\infty}^{\infty} \int_{0}^{2 \pi} \frac{\eta}{\eta^{2}+y^{2}} q(x, y) \mathrm{e}^{\mathrm{i} m x} \mathrm{e}^{-\mathrm{i} k x} \phi_{n}(y) \phi_{s}(y) \mathrm{d} x \mathrm{~d} y,
$$


where $(k, s, m, n)$ define the element in the block and $q(x, y)$ characterizes the block. The basis functions in $x$ and $y$ imply

$$
I_{A}(k, s, m, n) \approx \sum_{i=1}^{N_{x}} \sum_{j=1}^{N_{y}} w_{x}(k) w_{\xi}(s) q\left(x_{i}, \xi_{j}\right) \mathrm{e}^{\mathrm{i} m x_{i}} \mathrm{e}^{-\mathrm{i} k x_{i}} \cos \left(n \xi_{j}\right) \cos \left(s \xi_{j}\right),
$$

where $w_{x}(k)$, and $w_{\xi}(s)$ are the normalization weights, $x_{i}$ are the collocation points in the $x$-direction, and $\xi_{j}$ the inverse cosine of the collocation points $Y_{j}$. The summations are done using routines from the FFTW package.

The matrix $\boldsymbol{B}$ is a constant block-diagonal matrix, depending only on the parameters $\left(N_{e}, N_{x}, N_{y}\right)$. The $N_{e}$ diagonal blocks are identical, the elements of which are given by

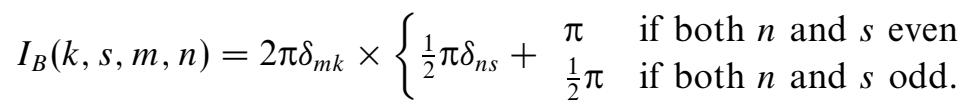

The eigenvalues of this system were computed using routines from the LAPACK package.

Since all base flows considered here are unbounded in $y$, the spectrum of the continuous operator is expected to contain both discrete and continuous components. Our physical interest is in the discrete part, which will represent large-scale compressible growth dynamics. It was therefore necessary to separate, numerically, the discrete spectrum from the much larger set of eigenvalues that are the discrete approximation to the continuous spectrum. This was done here by testing the convergence of each eigenvalue and eigenvector with increased resolution, the discrete spectrum showing rapid convergence to four figures or better, while the continuous spectrum converged much more slowly.

\section{Parallel base flows}

The numerical method was verified against known results for the stability of compressible parallel base flows: Sandham \& Reynolds (1990, 1991), Zhuang et al. (1990b), Lin (1953), Lessen et al. (1965). Two different base flows were investigated: the class of Crocco-Busemann (CB) profiles and a constant-density, parallel, hyperbolictangent velocity profile (CD). For the $\mathrm{CB}$ profiles, the Crocco-Busemann relationship is used to relate a parallel temperature profile to a parallel velocity profile. Here, we make the additional simplification of specifying a simple hyperbolic tangent profile, with a parameter $\omega_{h}$ set to fix the vorticity thickness

$$
\bar{u}(y)=\tanh \left(\omega_{h} y\right), \quad \bar{T}(y)=1+\frac{1}{2}(\gamma-1) M_{\infty}^{2} \bar{u}^{2}(y), \quad \delta_{\omega}=\frac{2}{\omega_{h}} .
$$

We choose $\omega_{h}=1$ giving $\delta_{\omega}=2$. The resulting profile is close, but not identical, to the true $\mathrm{CB}$ profile. The $\mathrm{CD}$ profile is homentropic, whereby its stability analysis admits a single equation for $\rho^{\prime}(x, y, z, t)$, used extensively in the Appendix.

The convective Mach number $M_{c}$, (Bogdanoff 1983; Papamoschou \& Roshko 1988), allows results from temporal and spatial stability analysis to be compared. For the type of flows used in this analysis $M_{c}=M_{\infty}$, implying that the two may be used interchangeably. Table 1 shows results from runs with $N_{y}=64$ and $N_{y}=256, \eta=1.5$, compared with results from Sandham \& Reynolds (1990). In the range $n=0-40$, the agreement of the Chebyschev coefficients, $\left(a_{n}, \boldsymbol{b}_{n}, c_{n}\right)$, from the different resolutions, is on the order of four figures, where they decay exponentially fast by four orders of magnitude. For values of $M_{\infty}$ above about 0.6, the most amplified modes become three-dimensional, in good agreement with Sandham \& Reynolds (1990, 1991). 


\begin{tabular}{cccc} 
& \multicolumn{3}{c}{$\sigma_{r}$} \\
\cline { 2 - 4 }$M_{c}$ & $N_{y}=64$ & $N_{y}=256$ & S\&R \\
0.01 & 0.1896791 & 0.1896792 & 0.189 \\
0.60 & 0.1175104 & 0.1175119 & 0.116 \\
1.20 & 0.0529660 & 0.0529666 & 0.053
\end{tabular}

TABLE 1. Calculated values of $\sigma_{r}$, computed at $\left(\alpha_{\max }, \beta_{\max }\right)$, compared at different resolutions for the CB profile. S\&R denotes Sandham \& Reynolds (1990).

$\begin{array}{ccc}\text { Case } & \epsilon & \kappa \\ \mathrm{A} & 0.0283 & 1.0001 \\ \mathrm{~B} & 0.2826 & 1.0100 \\ \mathrm{C} & 0.8871 & 1.1000\end{array}$

TABLE 2. The three representative values of the mass flux, $\epsilon$, used.

\section{Instabilities of the CSV}

We now consider the stability of the non-uniform CSV states. The base flow is given by numerical solutions to (2.2)-(2.3) obtained by MMP using a spectral method, and repeated here for the stability calculations. We emphasize that known CSV solutions have $0 \leqslant M_{\infty}<1$, even though they may contain embedded regions of locally supersonic flow. Thus our stability analysis is limited to non-uniform compressible shear flows with subsonic free streams. For the stability problem, there is a four-dimensional space of parameters comprising $\epsilon, M_{\infty}$ for the base flow and $\alpha$, $\beta$. Here we consider three representative values of $\epsilon$, shown in table 2, across a range of $M_{\infty}$. Figure 1 shows examples of the base flow at these values for $M_{\infty}=0.51$. Case A is a near-parallel flow and is represented well by the solution derived from the perturbation analysis in the Appendix. For case $\mathrm{C}$, the base flow is highly non-parallel with the velocities in the $x$ - and $y$-directions on the same order of magnitude. The coherent spanwise vortices have become compact, and the dilatation has risen by two orders of magnitude from case A. The continuation of case $\mathrm{C}$ terminates at $M_{\infty}$ just above 0.6. This is thought to be due to the presence of shocklets, which appear to decelerate the flow from supersonic conditions at the edge of the vortex, to the stagnation points between the vortex cores. Sandham \& Reynolds (1991) observed the appearance of shocks in two-dimensional unsteady simulations at similar $M_{\infty}$. This suggests that case $\mathrm{C}$ may provide the best model of the vortical structures in the compressible shear layer.

The spectral solutions reported are for $\eta=1.5$, and $\left[N_{x}, N_{y}\right]=([32,32],[32,64]$, $[64,32])$, with four-figure agreement found for the growth rates from the various resolution runs. The larger resolutions are the highest which could be achieved with available computing resources. It is necessary to scale $\left(\sigma_{r}, \alpha, \beta\right)$ with the vorticity thickness of the base flow,

$$
\delta\left(\epsilon, M_{\infty}\right)=-\frac{1}{\pi} \int_{0}^{2 \pi} \int_{0}^{\infty} y \omega(x, y) \mathrm{d} x \mathrm{~d} y .
$$

The scaling factors are given by $\delta\left(\epsilon, M_{\infty}\right) / \delta(\epsilon, 0)$, figure $2(a)$. 

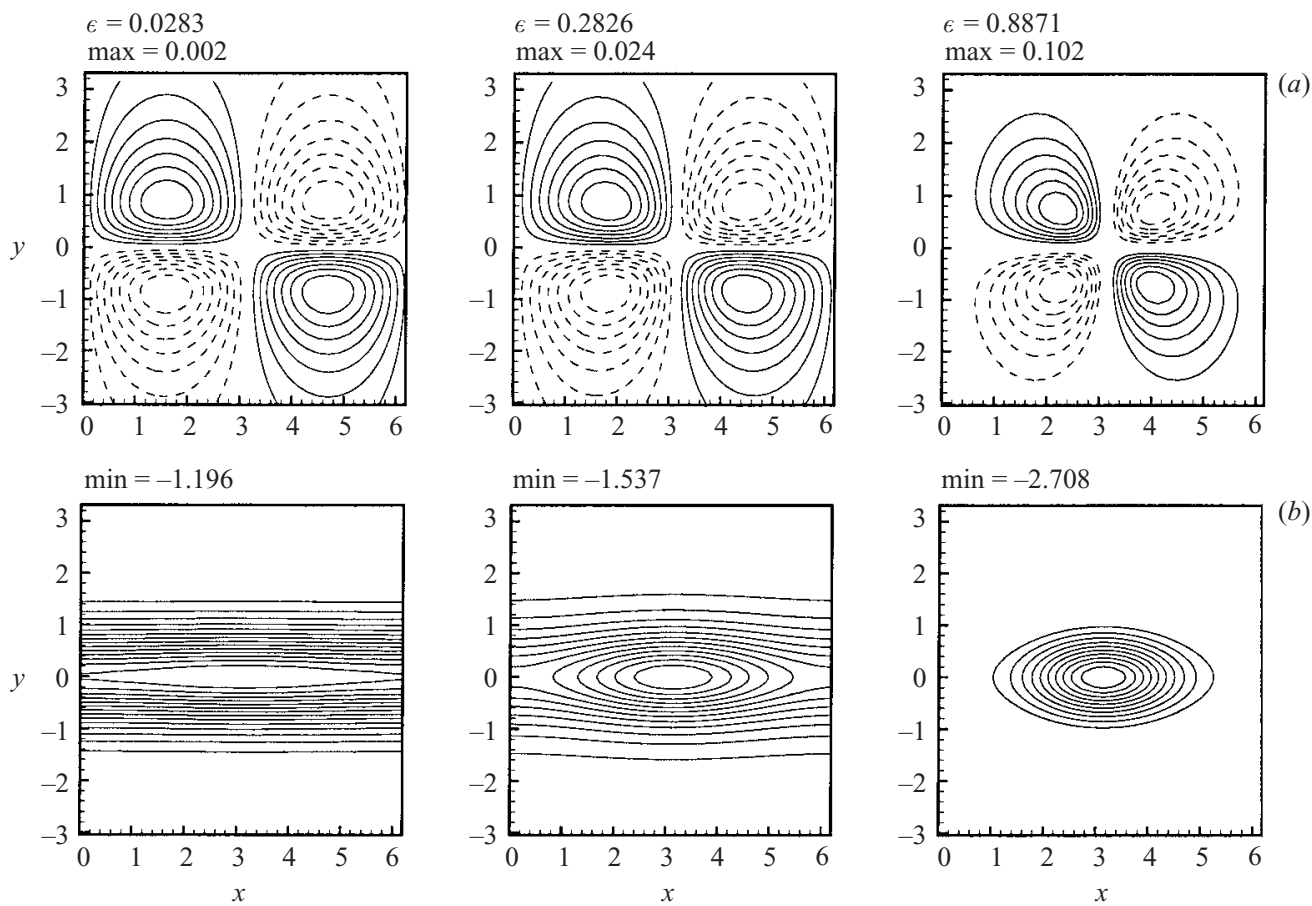

FiguRE 1. Examples of the base flow profiles for $M_{\infty}=0.51:(a) \nabla \cdot \boldsymbol{u}(x, y),(b) \omega(x, y)$. The maximum or minimum contour value is given for each profile; dashed lines indicate negative contours here and in subsequent similar figures.
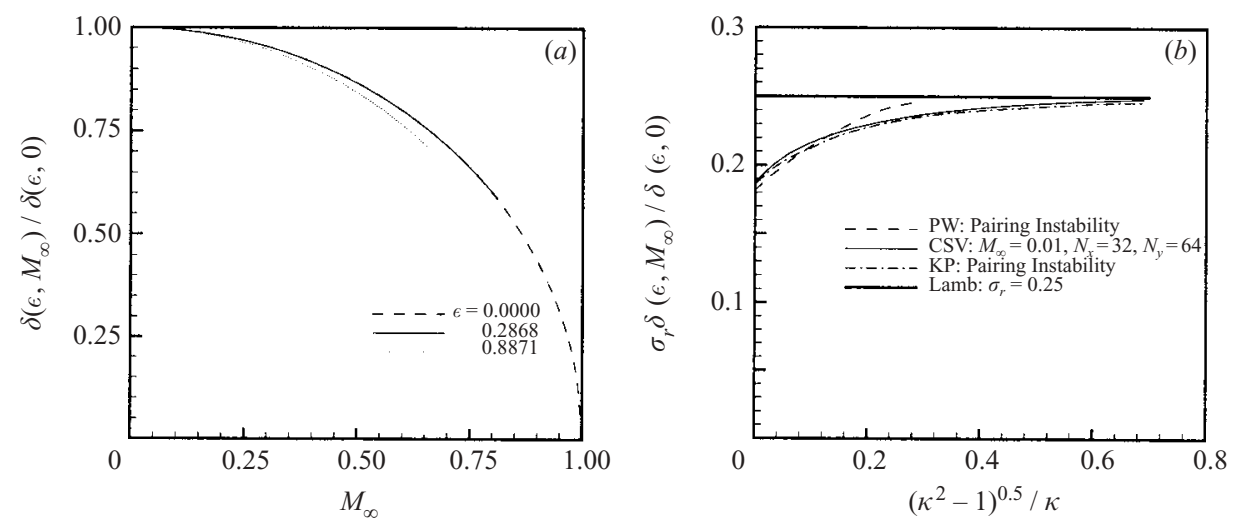

FIGURE 2. (a) Scaling factors determined from the vorticity thickness. The zero-mass-flux curve is given by $\left(1-M_{\infty}^{2}\right)^{1 / 2}$, an expression obtained from the perturbation analysis in the Appendix. (b) Comparison with Pierrehumbert \& Widnall (1982) and Klaassen \& Peltier (1989).

\subsection{Incompressible limit, $M_{\infty}=0$}

The stability algorithm may be run with $M_{\infty}=0.01$ for comparison with $\mathrm{PW}$ and Klaassen \& Peltier (1989, henceforth referred to as KP). We first discuss $\alpha=0.5$ (the first subharmonic) in which adjacent vortices in the base flow are displaced in opposite directions. The growth rates for $\alpha=0.5, \beta=0$ are shown in figure $2(b)$ as a function of core size parameter. The growth rates are real, increasing monotonically 

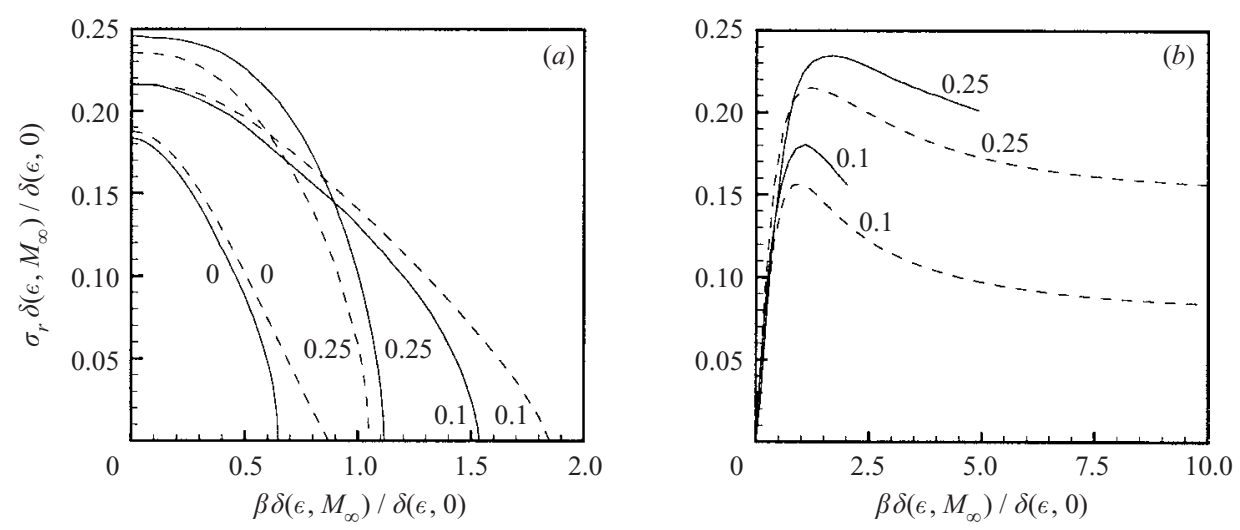

FIGURE 3. Solid lines: Pierrehumbert \& Widnall (1982), dashed lines: results from CSV runs with $M_{\infty}=0.01$. Labels represent the value of the vortex core size parameter. $(a)$ Helical pairing instability, $\alpha=0.5$. (b) Translative instability, $\alpha=0$.

as the core size parameter is increased toward the Lamb (1932) limit of $\sigma_{r}=0.25$ for a row of point vortices. Differences in resolution, $[32,64]$ here and $[4,6]$ for PW, account for the discrepancies in the growth rates. The agreement with KP, who used double the resolution of PW, is satisfactory. Also results very like PW were found when using their resolution. We find that growth rates close to the point-vortex limit are achieved at $\epsilon=1.696$, for which the two-dimensional pairing growth rate has risen to $\sigma_{r}=0.2482$. Modes with finite $\beta$ are referred to as helical pairing modes. Their growth rates are shown in figure 3(a) compared with PW. These modes have a short-wave cutoff in $\beta$, which implies that spanwise scales with $\beta \delta_{\omega}>$ const do not amplify as they advect downstream.

Our final comparison with PW is done for the translative instability for which $\alpha=0$. Perturbations then have the same $x$-periodicity as the base flow from which it follows that this mode is not an extension of any parallel flow instability. The growth rates are shown in figure 3(b). When $\epsilon \rightarrow 0$ (parallel base flow), the growth rates fall identically to zero. In the incompressible limit, the maximum growth rate of this instability, for fixed $\epsilon$, is just smaller than that of the two-dimensional pairing instability.

\subsection{Compressible instabilities}

\subsubsection{Two-dimensional subharmonic instabilities}

We first discuss two-dimensional modes with $\beta=0$. Generally, the discrete spectrum consisted of three distinct real eigenvalues. The growth rates of the largest of these eigenvalues are shown in figure 4. This eigenvalue always attains its maximum value for the subharmonic mode $\alpha=0.5$, which seeds the pairing instability. To compute the mode shape, the eigenvector is first normalized, and multiplied by the $\mathrm{e}^{\mathrm{i} \alpha x}$ phase factor. Each eigenmode is chosen to be purely real. The sign and absolute magnitude of these modes are arbitrary, but the magnitude of the perturbation variables relative to each other can be important, as it is an indicator of the dominant mechanisms by which the linear instability acts in any given area of the parameter space. Figure 5 shows contour plots of selected eigenmodes from case $\mathrm{C}$ runs. For case A and B runs, the smaller values of $\epsilon$ produce thin flat base-flow vortices, figure 1. This is reflected in the eigenmode structure for these runs, which show similar features to the case $\mathrm{C}$ modes. As with parallel base flows, the density perturbation is unimportant for the low Mach number runs, being four orders of magnitude smaller than either of the velocity 

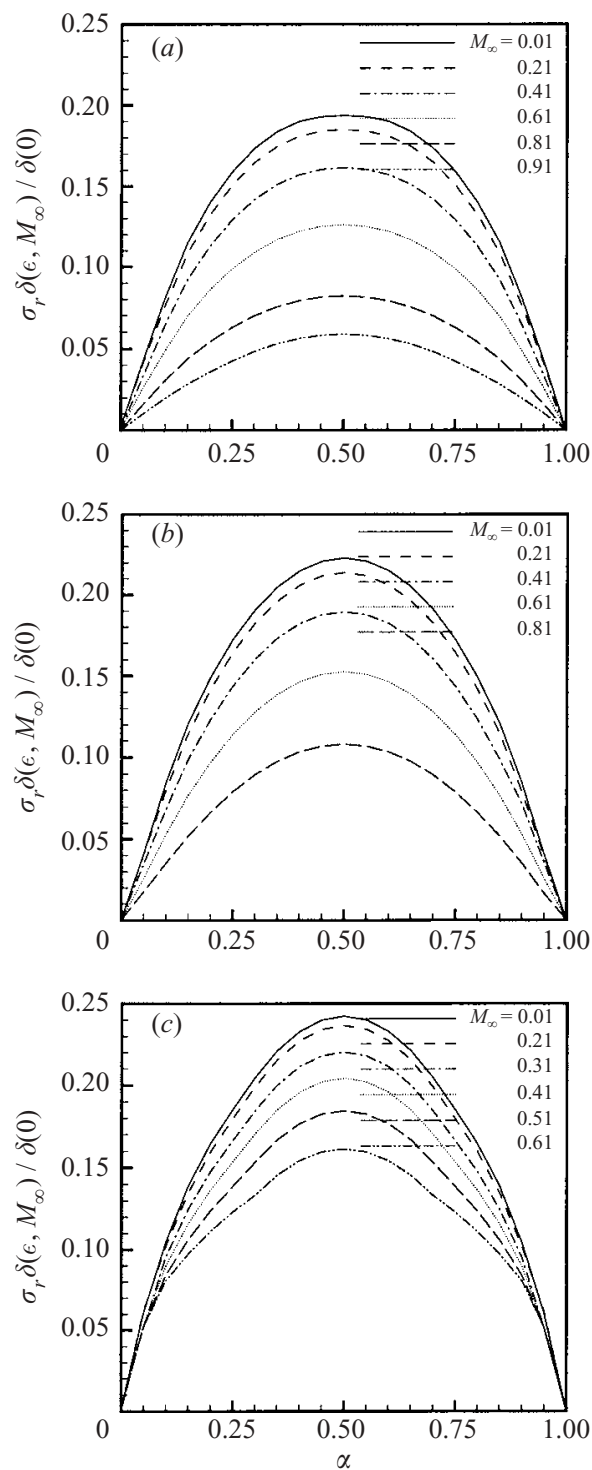

FIGURE 4. Pairing instability growth rate: $(a) \epsilon=0.0283,(b) \epsilon=0.2826,(c) \epsilon=0.8871$.

perturbations. The density eigenmodes keep the same basic shape across the Mach number range, but by $M_{\infty}=0.61$ these modes have increased by four orders of relative magnitude.

Two examples of vorticity eigenmodes (obtained from the velocity eigenvectors) are shown in figure 5 . These resemble a skewed vortex dipole, which become flattened and elongated as $M_{\infty}$ increases. This eigenmode has a nodal line which runs through the centre of the unperturbed vortex. The angle that this nodal line makes with the positive $x$-axis is labelled $\phi_{1},-90^{\circ}<\phi_{1}<0^{\circ}$. The presence of a sloped nodal line implies that this eigenmode initiates the clockwise rotation of the vortices at $x=\pi$ and $x=3 \pi$ about each other, which ultimately leads to pairing. The more negative $\phi_{1}$, the more effective the eigenmode is at initiating a pairing event. The angle between 

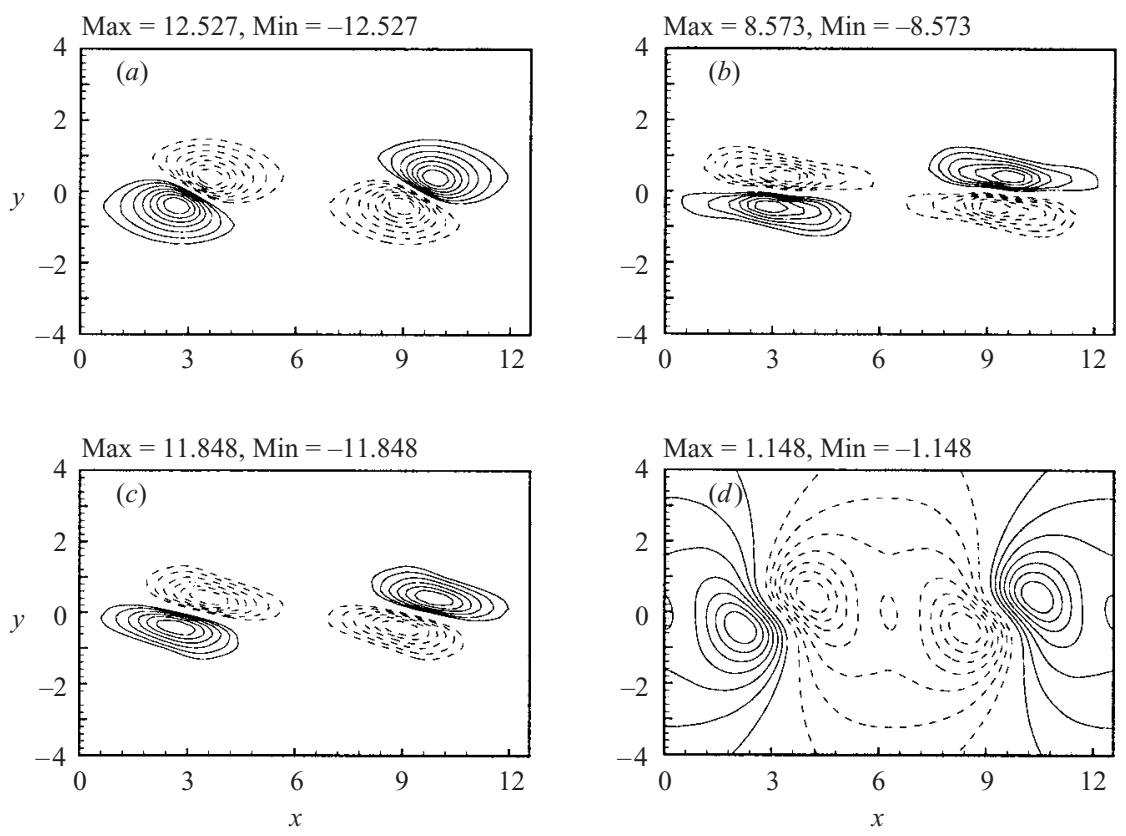

FIGURE 5. Two-dimensional pairing instability, $\epsilon=0.8871$. (a) $M_{\infty}=0.01$, contours of spanwise vorticity. (b) $M_{\infty}=0.61$, contours of spanwise vorticity. (c) $M_{\infty}=0.61$, contours of dilatation. (d) $M_{\infty}=0.61$ contours of density.

\begin{tabular}{cccc} 
& \multicolumn{3}{c}{$\left|\phi_{1}\right|($ deg. $)$} \\
\cline { 2 - 4 }$M_{\infty}$ & case A & case B & case C \\
0.01 & 2.5 & 17.1 & 28.1 \\
0.61 & 0.8 & 5.5 & 6.1 \\
0.81 & 0.5 & 3.2 & -
\end{tabular}

TABLE 3. Values of $\left|\phi_{1}\right|$ for the various mass fluxes investigated.

the line connecting the maximum negative and positive values of vorticity and the nodal line is denoted $\phi_{2}$, and is a measure of the skewness of the eigenmode. Over the range of $M_{\infty}$ investigated, $\phi_{2}$ remains almost constant for each of the different runs: $\phi_{2} \sim 28^{\circ}$ for case $\mathrm{A}, \phi_{2} \sim 50^{\circ}$ for case $\mathrm{B}$, and $\phi_{2} \sim 68^{\circ}$ for case C. In contrast, increasing $M_{\infty}$ has a dramatic effect on $\phi_{1}$, with the slope of the nodal line becoming less negative as $M_{\infty}$ increases; see table 3 . Thus, increasing compressibility not only damps the growth rate of the pairing instability, but it also decreases the eigenmode's effectiveness in initiating the pairing process, as seen in figure 6.

\subsubsection{A parallel instability}

The next most vigorously amplified instability is largely independent of mass flux. Its growth rate behaviour and that of an instability to a parallel $C D$ profile show remarkable similarity, figure 7. Indeed, growth rates from case A and B runs agree with two- and three-dimensional CD growth rates to within four figures. The eigenmode structure, less the $\mathrm{e}^{\mathrm{i} \alpha x}$ prefactor, is independent of $x$, and bears a striking likeness 

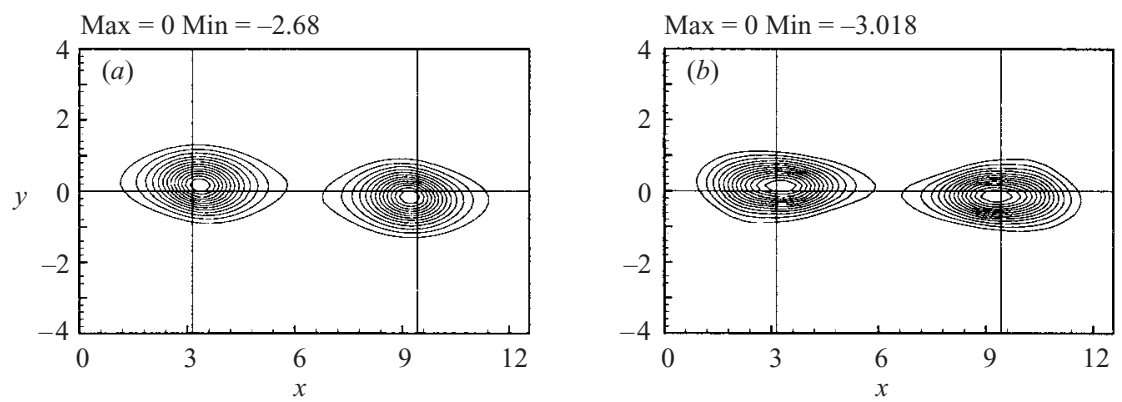

FIGURE 6. Base flow vorticity in the spanwise direction plus an eigenmode. The eigenmode is computed at a time $t_{1}$ such that the maximum value of vorticity of the eigenmode is $20 \%$ that of the vorticity in the base flow, $t_{1} \propto \sigma_{r}^{-1}$. The line intersections mark the centre of an unperturbed vortex. $(a) \epsilon=0.8871, M_{\infty}=0.01$. $(b) \epsilon=0.8871, M_{\infty}=0.61$.
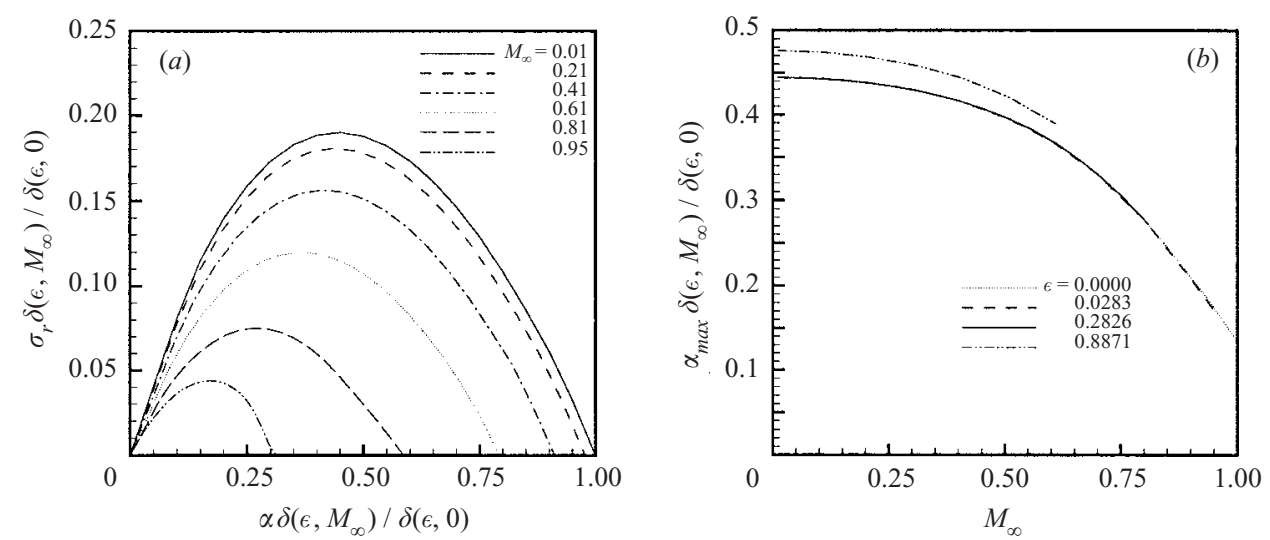

FiguRE 7. (a) Two-dimensional parallel instability growth rates computed at different mach numbers for $\epsilon=0.0283$. (b) $\alpha_{\max } \times \delta\left(\epsilon, M_{\infty}\right) / \delta(\epsilon, 0)$ vs $M_{\infty} ; \epsilon=0$ represents the parallel CD base flow.

to the structures arising from a CD base profile. This suggests that it corresponds to an instability occurring on the parallel shear layer, and it is therefore denoted the parallel instability. Its presence implies that even after nonlinear processes have caused the primary roll-up of the parallel flow, the instability which initiated the roll-up remains active and relatively unaltered, except that it becomes subdominant to the more unstable pairing instability.

The real part of the third largest eigenvalue decays rapidly as either $M_{\infty}$ or $\epsilon$ is increased, being extremely weakly amplified for case $\mathrm{C}$ runs. Usually, it maximizes at $\alpha=0.5$, and so may be considered subharmonic. Over a certain range of the base flow parameters it becomes bimodal, figure 8. Its eigenmodes indicate that it tends to alter the strength of neighbouring vortices, enhancing one while diminishing another. It may be linked to the draining instability discovered by KP, and can be seen as an aid to the pairing process: Winant \& Browand (1974). The eigenmodes show some small-scale structure, indicating that these higher-order modes are sensitive to numerical inaccuracy and inaccuracy in the inviscid physical model. Due to its weak amplification at higher values of $\epsilon$ and $M_{\infty}$, the three-dimensional properties of this instability are not investigated exhaustively. 

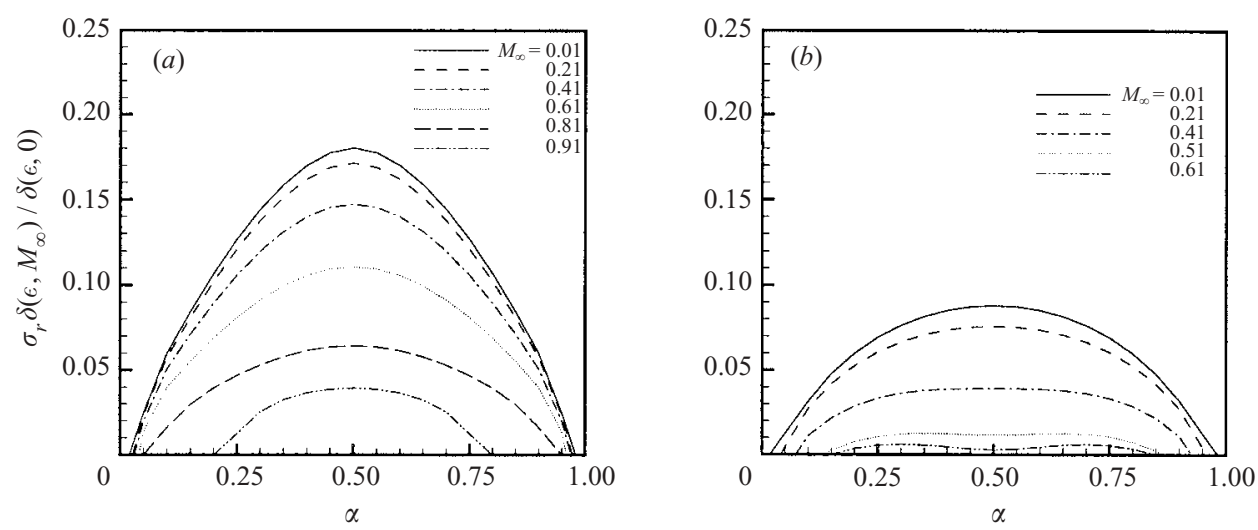

FIGURE 8. Draining instability; $(a) \epsilon=0.0283,(b) \epsilon=0.2826 . \sigma_{r} \times \delta\left(\epsilon, M_{\infty}\right) / \delta(\epsilon, 0)$ vs $\alpha$.

\subsubsection{Three-dimensional subharmonic instabilities}

We now discuss results obtained for the pairing and parallel instabilities, the two most unstable in two dimensions, for non-zero values of $\beta$. Relevant growth rates are shown in figure 9. The parallel mode again behaves as if it were an instability on a parallel shear layer, and for $M_{\infty}<0.6$ is most unstable in the two-dimensional limit. The pairing mode is also most unstable in the two-dimensional limit below some critical value of $M_{\infty}$, which decreases as $\epsilon$ is increased. The pairing mode remains subharmonic, with $\alpha_{\max }=0.5$ for all values of $\beta$ and $M_{\infty}$. Note that for case $\mathrm{C}$ runs, above the transitional value of $M_{\infty}$, for $\beta<0.25$ the growth rate curves are relatively flat, indicating that there is no single dominating spanwise wavelength. In contrast to the parallel instability, the short-wave cutoff for the pairing mode shows strong dependence on both $M_{\infty}$ and $\epsilon$. This may be due to thin flat vortex-like structures, present in low- $\epsilon$ base flows, supporting small-scale instabilities, which are damped by the stabilizing effect of self-induced rotation of the more compact vortex cores present in high- $\epsilon$ base flows (Rosenhead 1930).

The $z$-dependence of the vorticity eigenmodes may be deduced from the symmetry properties of the governing equations. The anti-nodal points of the spanwise vorticity are located at $\beta z=0, \pm \pi, \pm 2 \pi \ldots$, which correspond to the nodal points of both the streamwise and transverse modes. At low Mach numbers, $M_{\infty}<0.4$, the spanwise vorticity structure is similar to figure $5(a)$, with the difference that $\phi_{1}$ decreases slightly as $\beta$ increases. PW suggested that the helical pairing mode would promote localized pairing of neighbouring vortices. This would lead to phase dislocations in the spanwise direction (Chandrsuda et al. 1978), and the generation of coherent three-dimensional structures connecting the spanwise vortices like those seen in plan views of low Mach number mixing layers (Clemens \& Mungal 1995). For $M_{\infty}>0.4$ the spanwise vorticity assumes a wavy structure, figure $10(a)$. At $\beta z=0$, the vortex at $\pi$ is no longer shifted up and to the right, so that localized pairing may occur; rather it now moves up and slightly to the left. Thus, the base flow tends to resist the action of the linear instability. Upon consideration of the streamwise vorticity eigenmode, it is plausible that the perturbation of figure 10 would lead to a hairpintype structure, with the heads of the hairpin located at $\beta z=0,2 \pi \ldots$ and the legs at $\beta z=\pi / 2,3 \pi / 2 \ldots$ (Sandham \& Reynolds 1991). The fact that these structures are not readily identifiable in mixing layer experiments at low Mach numbers may be due to a combination of effects. The small relative magnitude of the streamwise 

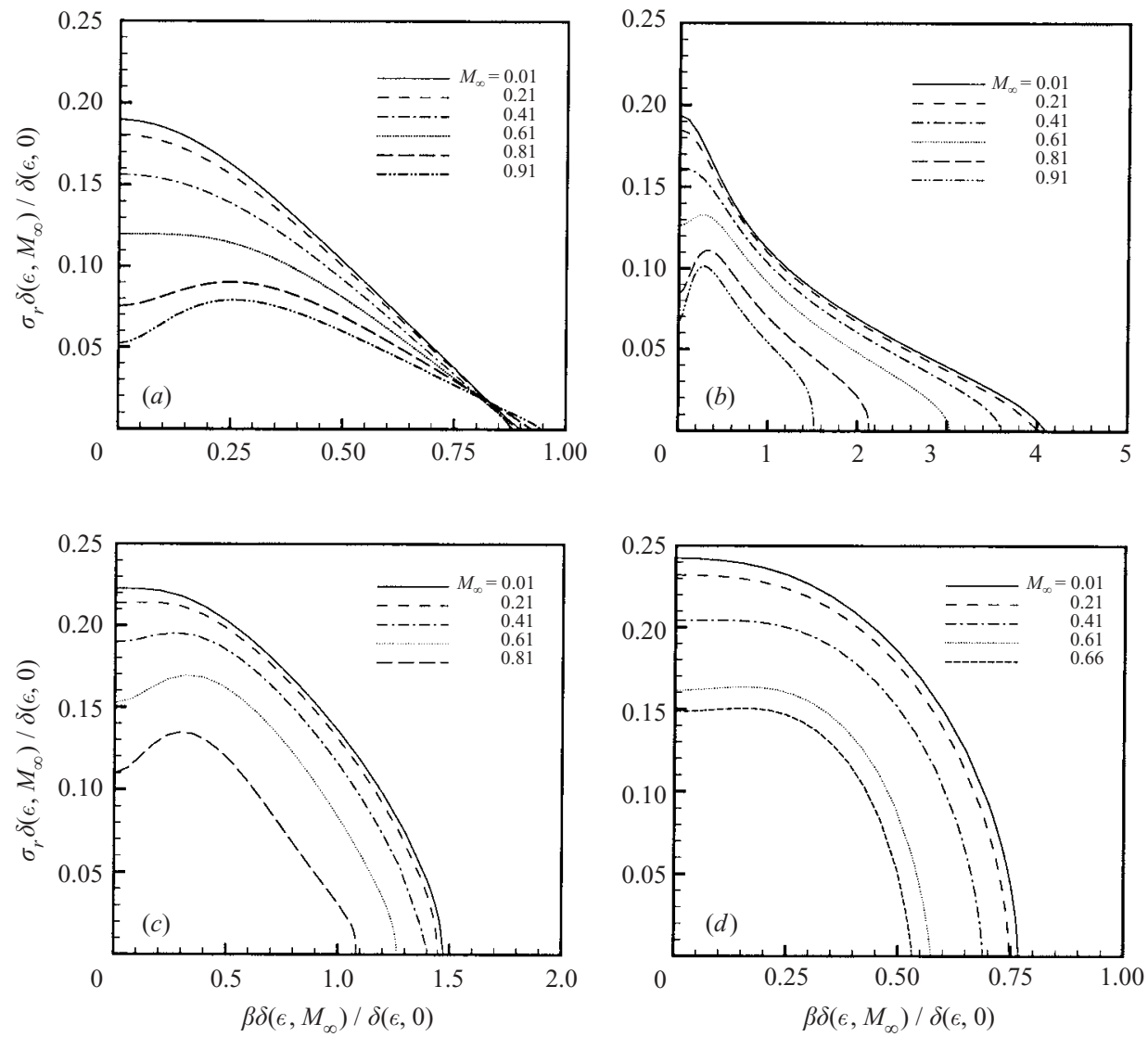

FIGURE 9. Scaled growth rates versus spanwise wavenumber. The streamwise wavenumber is held at its two-dimensional $\alpha_{\max }$ value, which depends on $M_{\infty}$, as $\beta$ is varied. (a) Parallel instability, $\epsilon=0.0283$. (b) Helical pairing instability, $\epsilon=0.0283$. (c) Helical pairing instability, $\epsilon=0.2826$. $(d)$ Helical pairing instability, $\epsilon=0.8871$.

vorticity eigenmode, combined with the short-wave cutoff in $\beta$, implies that the instability would saturate before nonlinear processes take over. Therefore, as with two-dimensional subharmonic modes, as $M_{\infty}$ increases, the subharmonic instabilities do not trigger interactions between neighbouring vortices.

\subsubsection{Three-dimensional fundamental modes}

These modes have the same periodicity in the streamwise direction as the base flow. For finite $\beta$ the spanwise vorticity eigenmode is anti-symmetric about its centre, and remains so even as $M_{\infty}$ is increased; figure 11 . This implies that the instability causes a net translation of the vortex cores, up and to the right at spanwise locations $\beta z=0, \pm 2 \pi \ldots$, rather than a bulging. A fundamental mode with no spanwise variation is neutrally stable, and shifts the vortex row an infinitesimal distance. For these reason, PW labelled this mode the translative instability.

The linear incompressible mechanism by which regions of two-dimensional elliptical streamlines can generate three-dimensional flows is denoted the elliptical instability. It is localized in the vortex core, with growth rates tending to a finite value as the wavelength along the vortex axis tends to zero: Pierrehumbert (1986), Bayly (1986). In viscous flows this inviscid mechanism leads to real vortex instability, with a 

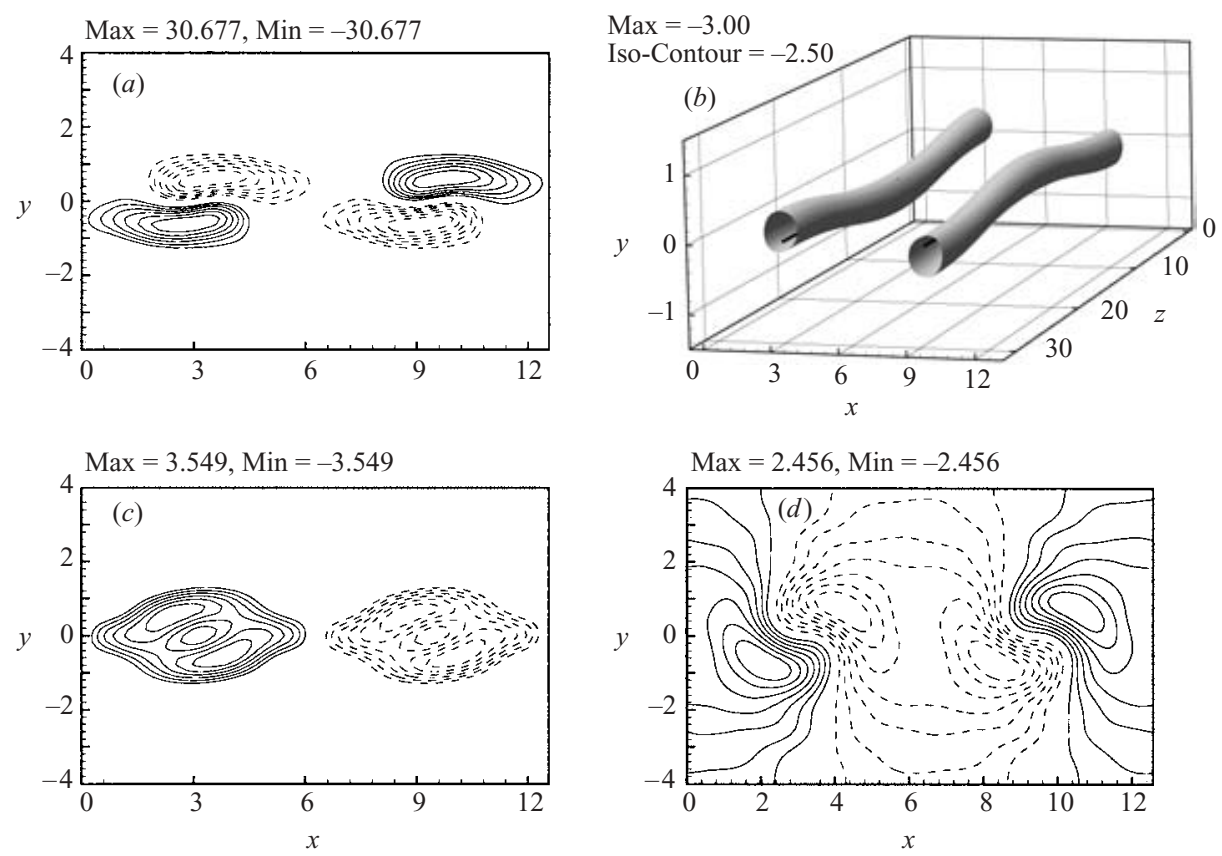

FIGURE 10. Helical pairing eigenmodes, $\beta=\beta_{\max }=0.211, \epsilon=0.8871, M_{\infty}=0.61$. (a) Spanwise vorticity. (b) Base vorticity plus spanwise perturbation with amplitude chosen so that its max is $20 \%$ that of the base flow. (c) Streamwise vorticity. (d) Density.
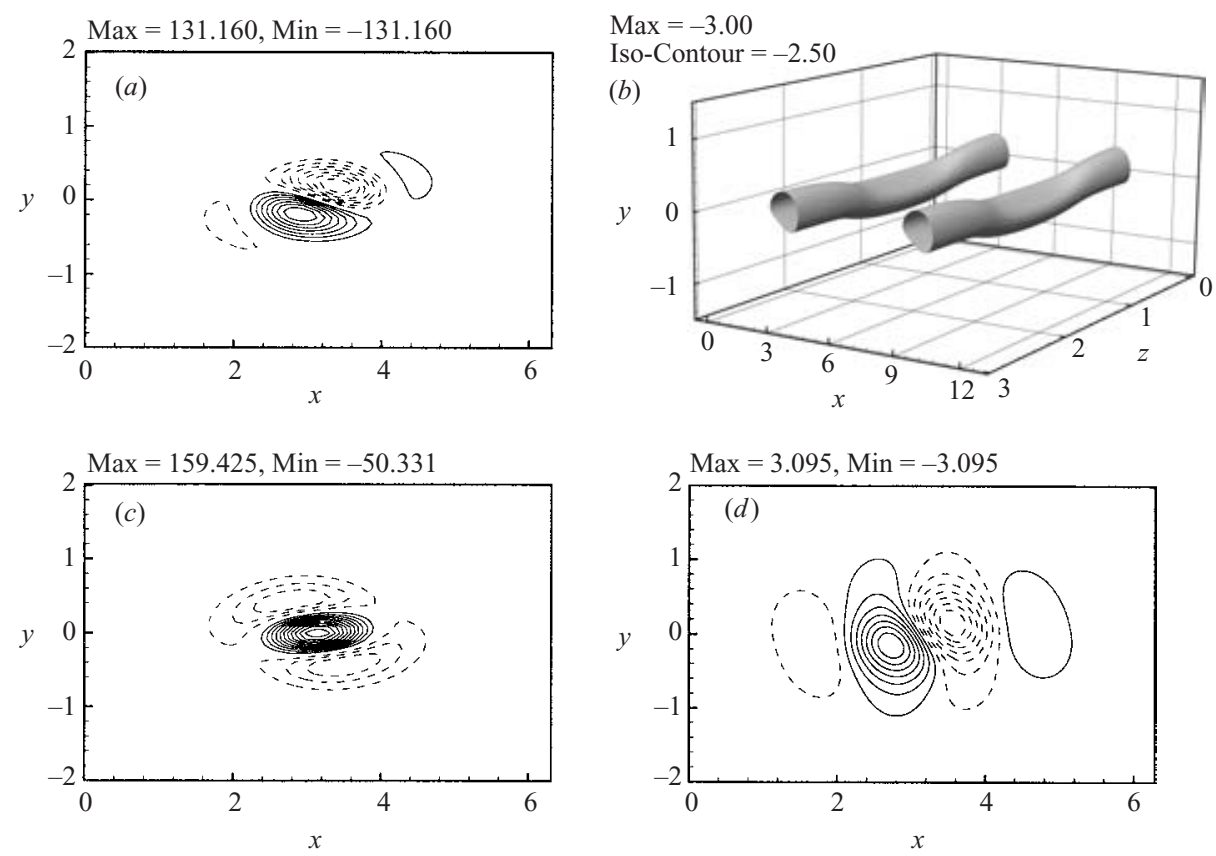

FIGURE 11. Translative instability eigenmodes, $\beta=\beta_{\max }=2.226, M_{\infty}=0.61, \epsilon=0.8871$. A full period is shown in all cases. (a) Spanwise vorticity perturbation. (b) Base vorticity plus spanwise perturbation with amplitude chosen so that its max is $20 \%$ that of the base flow. (c) Streamwise vorticity perturbation. $(d)$ Density eigenmode. 

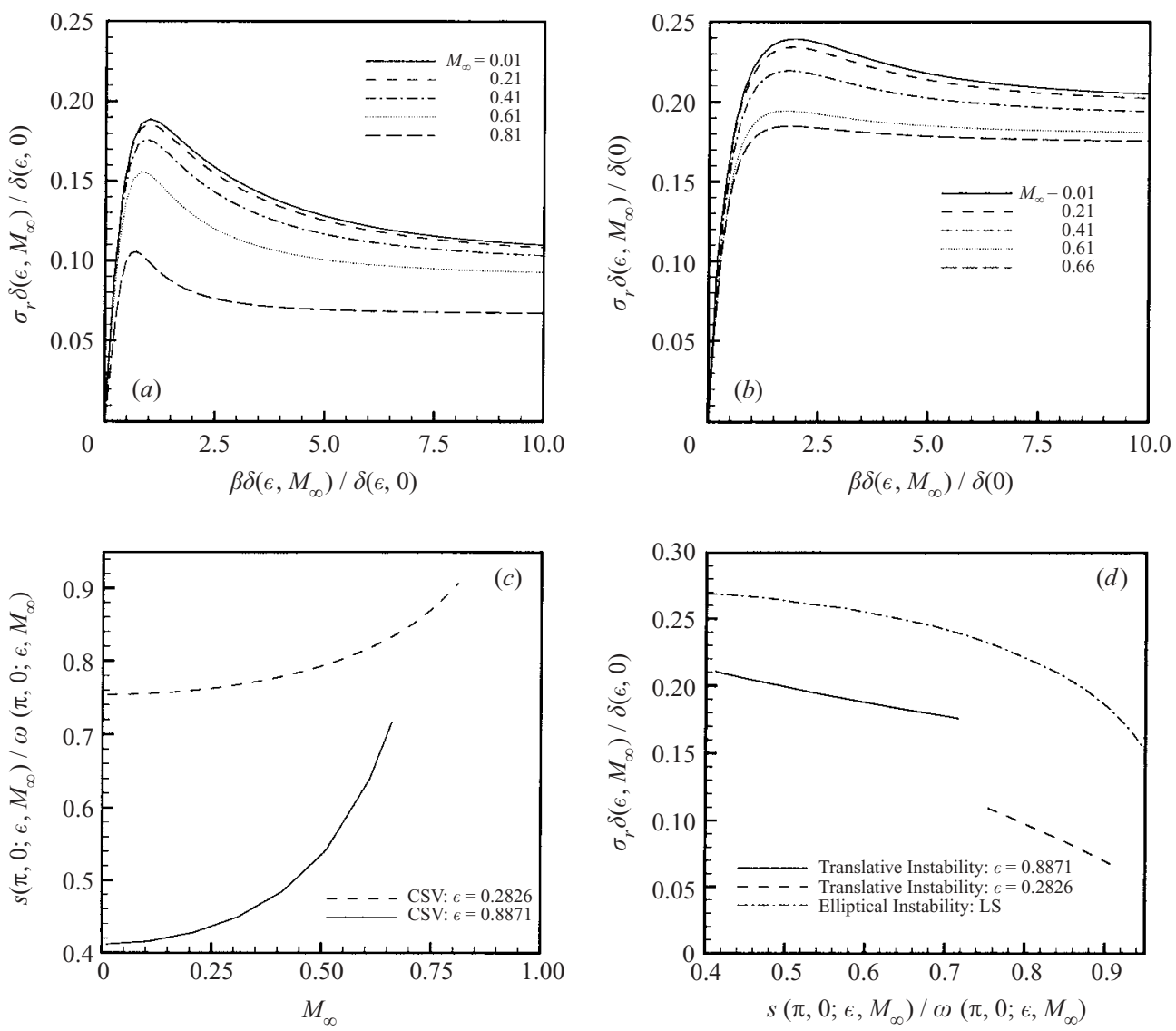

FIGURE 12. Three-dimensional translative instability growth rates, computed at various values of $M_{\infty}, \alpha=0$. (a) $\epsilon=0.2826,(b) \epsilon=0.8871,(c)$ Normalized vortex core strain vs $M_{\infty}$. (d) Growth rates of the translative instability, where $\beta \times \delta\left(\epsilon, M_{\infty}\right) / \delta(\epsilon, 0)=10$, compared to those from an elliptical instability plotted against the normalized strain rate, LS denotes Landman \& Saffman (1987).

short-wavelength cutoff imposed by the action of viscosity (Landman \& Saffman 1987). The physical mechanism of instability is one of vortex stretching (Waleffe 1990), and may exist in a relatively unaltered state in compressible subsonic gases, where the growth rates and eigenmodes depend only on the local velocity gradient tensors of the basic flow (Lifschitz \& Hameiri 1991). Thus, the translative mode structure, figure 11, and growth rates, figure 12 , link it to an instability of the elliptical type. It is unique to non-parallel flows, for parallel flows it is not present, while for near parallel flows, case A computations, it is very weakly amplified.

The normalized strain, $s(\pi, 0) / \omega(\pi, 0)$, of the unperturbed vortex cores yields a measure of the local ellipticity of the CSV streamlines. A value of zero gives locally circular streamlines, while a value of one implies infinite ellipticity, that is a locally plane Couette flow. The agreement between growth rates from the translative instability, parameterized by the normalized strain, and from pure elliptical flows is marginal, figure $12(d)$. However, this may explain why the translative instability shows little damping with increased levels of compressibility. For case $\mathrm{C}$ runs, with $M_{\infty}>0.2$, this mode represents the most unstable perturbation to the CSV. 
PW speculated that the deposition of streamwise vorticity, by the eigenmodes and a tilting process induced by the base flow, would lead to the creation of counterrotating streamwise vortices. Sandham \& Reynolds (1991) simulated a translative-type instability and showed how the straining field of this type of instability can pull the vorticity into the braid regions, leading to the formations of streamwise vortices (Lin \& Corcos 1984). A feature necessary for a streaky streamwise structure to occur is a single dominant spanwise wavenumber emerging from a random perturbation. As $M_{\infty}$ increases figure 12 implies that this scenario is highly unlikely. It is not clear that a superposition of many translative instabilities, with the same growth rate but different spanwise wavelengths, could produce a coherent three-dimensional structure.

\subsection{Comparison with experiment}

In incompressible flows, large eddies play an important role in both entrainment and determining the growth rates of the shear layer through pairings and amalgamations. For the CSV, the pairing-type instabilities maximize at subharmonic streamwise wavelengths over the entire $M_{\infty}$ range. However, their ability to trigger interactions between neighbouring vortices becomes very much reduced as the convective Mach number is increased. This is consistent with visualizations from Papamoschou \& Bunyajitradulya (1996), who for $M_{c}>0.5$ find no evidence of pairing in their compressible shear layers. They also speculate that the lack of organization in both the side and plan views suggests the coexistence of both two- and three-dimensionalities in the flow. Again this is consistent with the picture obtained from the CSV, where the growth rates of the pairing, helical pairing and translative modes are very similar over much of the Mach number range.

Stability analysis of parallel base flows suggest that at a given Mach number there is one spanwise wavelength which has maximum amplification. However, the plan views from Papamoschou \& Bunyajitradulya (1996) show that the chaotic patterns reveal every possible oblique angle to the free-stream flow. A possible explanation for this may be found in figures $13(a), 9$ and 12. These suggest that the range of spanwise wavenumbers with similar growth rates is quite large. Indeed for the higher Mach number case $\mathrm{C}$ runs the growth rate for the translative instability reduces by as little as $5 \%$ from its maximum value as the spanwise wavenumber increases by a factor of five.

In order to compare data from different experiments, the growth rates obtained must be normalized by the growth rates of an incompressible shear layer with the same density and velocity ratios. A variety of models, containing one or more free parameters, have been used for this purpose: Bogdanoff (1983), Ragab \& Wu (1989), Clemens \& Mungal (1995), Slessor et al. (2000). These different normalization methods, combined with a non-injective relationship between density ratio and convective Mach number, lead to substantial scatter in experimental growth rate data. The normalized growth rate trends for the three different mass fluxes investigated, as a function of $M_{c}$, are shown with results from various experimental investigations in figure 13. At any given $M_{c}$, the scaled growth rates from the CSV stability calculation lie in the mid to high range of the various experimental results. We remark that the present study yields temporal linear growth rates, whereas experiments measure the spatial growth of the mixing layer. Our results and those of Papamoschou \& Bunyajitradulya (1996) indicate a distinct lack of interaction between eddies in supersonic shear layers. Their slow evolution may be due a combinations of decreased growth rates and this lack of interaction. Whatever the reason it is difficult 

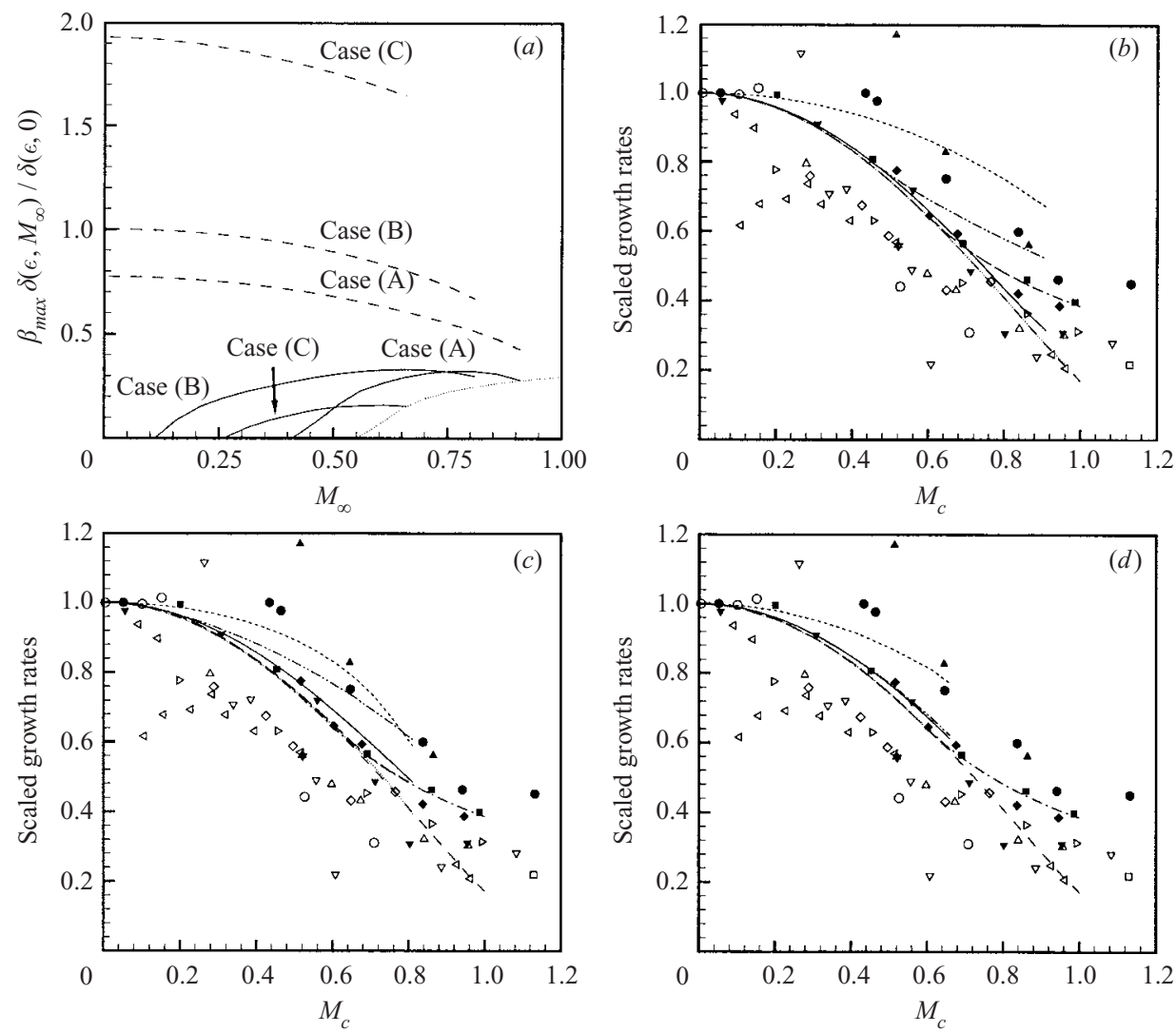

Figure 13. (a) $\beta_{\max } \times \delta\left(\epsilon, M_{\infty}\right) / \delta(\epsilon, 0)$ vs $M_{\infty}$. Solid lines represent the three-dimensional helical pairing instability. Dashed lines represent the three-dimensional translative instability. Dotted line for the three-dimensional parallel instability, $\epsilon=0$. (b) Case A. (c) Case B. (d) Case C. In $(b),(c)$ and $(d), \square$, Sirieix \& Solignac (1966); $\triangle$, Chinzei et al. (1986) (S \& CM); $\nabla$, Papamoschou \& Roshko (1988); \, Goebel \& Dutton (1991) (S); $\triangleleft$, Hall et al. (1993); $\diamond$, Clemens \& Mungal (1995); O, Slessor (1998); ם, Goebel \& Dutton (1991) (CM); Samimy \& Elliott (1990); $\mathbf{\nabla}$, Papamoschou \& Roshko (1986) •, Chinzei et al. (1986) (RW); Papamoschou (1986), dashed: CD two-dimensional modes, dash-dot: CD three-dimensional modes, solid: CSV two-dimensional pairing, dotted: CSV two-dimensional parallel, dashdot-dot: CSV three-dimensional helical pairing, long-dash: CSV three-dimensional parallel, short-dash: CSV three-dimensional translative. Initials after the experimentalists name, indicate by whom the results have been normalized: S, Slessor; CM, Clemens \& Mungal; RW, Ragab \& Wu.

to see their importance in governing the entrainment process in supersonic shear layers.

\section{Conclusions}

In the Appendix, we show that the two-dimensional neutral-stability wavenumber of a parallel shear flow in a constant-temperature compressible perfect gas is a stability bifurcation point where, at given free-stream Mach number, the solution branch corresponding to the CSV begins. This establishes a link between the linear stability of a class of parallel shear flows with tanh-velocity profiles in a compressible fluid and 
this special class of steady solutions to the Euler equations. It also partially motivates the extension of the theory of stability of plane parallel flows to include the stability of the spatially non-uniform CSV states themselves. This has been done here using a spectral-collocation method. As a physical model for the dynamics of compressible shear layers, the CSV structure is not without limitations, principally that for a fixed mass flux within a vortex core the homentropic solution branch terminates at a subsonic free-stream Mach number. Thus, while the CSV state apparently cannot be extended to supersonic free-stream flow, it nonetheless provides a useful base state for assessing the effect of compressibility on the stability properties of non-uniform compressible flows.

Three main classes of instabilities on the CSV were investigated: subharmonic, translative and a new parallel mode, each within the parameter space of the free-stream Mach number, the finite mass flow inside a closed vortex core and the wavenumber space of the perturbations. For any value of spanwise wavenumber it was found that the largest of the eigenvalues maximizes at either subharmonic or fundamental streamwise frequencies. The parallel instability, which might be interpreted physically as having initiated a primary roll-up producing a CSV-like structure, remains active and relatively unaltered. The persistence of this instability for the strongly nonlinear CSV flows may explain the success of linear growth rates, obtained from parallel shear flows, in postdicting experimentally observed growth rates in the compressible turbulent mixing layer.

In agreement with Pierrehumbert \& Widnall (1982), we found that for low Mach numbers the subharmonic mode has its greatest growth rate for eigenmodes with no spanwise variation, where it can be linked to an instability of the pairing type. As the Mach number increases this perturbation becomes three-dimensional and the 'term pairing' instability no longer applies, since it can no longer be interpreted as an initiating mechanism for interactions between neighbouring vortices. This is in agreement with experimental observations that the structures in compressible shear layers are largely inert. Not only do the subharmonic instabilities lose their ability to pair neighbouring vortices at higher Mach numbers, but this instability becomes subdominant to the more vigorous translative instability. The translative instability shows a broadband nature with respect to spanwise wavenumbers. This can be interpreted as being compatible with experimental observations, where structures at every possible oblique angle are observed.

We remark that the two-dimensional continuations of the finite-mass-flux CSV from a parallel flow, at fixed Mach number, is not unique. In particular a continuation from a three-dimensional neutral stability point is possible since the relevant stability curves do not terminate when the free-stream Mach number becomes supersonic. If such a continuation were admissible it may enable the construction of vortical, three-dimensional, globally supersonic solutions to the steady compressible Euler equations. Finally, the growth of non-homentropic disturbances to non-parallel base flows may be important. These may be investigated using a CSV constructed using a homenthalpic continuation to finite $M_{\infty}$ of the incompressible Stuart vortex. The entropy equation does not decouple from the system represented by (3.3)-(3.7), which may be physically relevant if the initial disturbances to experimental compressible shear layers were not approximately homentropic.

This work was supported by the Academic Strategic Alliances Program of the Accelerated Strategic Computing Initiative (ASCI/ASAP) under subcontract no. B341492 of DOE contract W-7405-ENG-48. 


\section{Appendix. Small-mass-flux limit: $\epsilon \ll 1, M_{\infty}$ finite}

In this Appendix we analyse the homentropic CSV equations, (2.2) and (2.3), at finite $M_{\infty}$ for $\epsilon \ll 1$. These equations are perturbed about a parallel constant-density profile of form to be determined from the analysis. This small-mass-flux solution is shown to coincide with neutrally stable perturbations to a CD base profile, Blumen (1970). Thus, the connection of the CSV to linearized-stability theory may be established. Numerical solutions of the CSV equations, following MMP, suggest an expansion of the form

$$
\begin{gathered}
\psi\left(x, y ; M_{\infty}, \epsilon\right)=\psi_{0}\left(y ; M_{\infty}\right)+\epsilon \psi_{1}\left(x, y ; M_{\infty}\right)+O\left(\epsilon^{2}\right), \\
\rho\left(x, y ; M_{\infty}, \epsilon\right)=1+\epsilon \rho_{1}\left(x, y ; M_{\infty}\right)+O\left(\epsilon^{2}\right), \\
\mu\left(M_{\infty}, \epsilon\right)=\mu_{0}\left(M_{\infty}\right)+O\left(\epsilon^{2}\right),
\end{gathered}
$$

where $\mu_{0}\left(M_{\infty}\right)$ is to be determined. On substitution into (2.2) and (2.3), it is found that

momentum $O(1): \frac{\mathrm{d}^{2} \psi_{0}}{\mathrm{~d} y^{2}}=\mathrm{e}^{-2 \mu_{0} \psi_{0}}$,

momentum $O(\epsilon): \quad \nabla^{2} \psi_{1}+2 \mu_{0} \mathrm{e}^{-2 \mu_{0} \psi_{0}} \psi_{1}=2 \mathrm{e}^{-2 \mu_{0} \psi_{0}} \rho_{1}+\frac{\mathrm{d} \psi_{0}}{\mathrm{~d} y} \frac{\partial \rho_{1}}{\partial y}$,

enthalpy $O(1): \quad\left(\frac{\mathrm{d} \psi_{0}}{\mathrm{~d} y}\right)^{2}=1-\frac{1}{\mu_{0}} \mathrm{e}^{-2 \mu_{0} \psi_{0}}$,

enthalpy $O(\epsilon):\left(\frac{1}{M_{\infty}^{2}}-\left(\frac{\mathrm{d} \psi_{0}}{\mathrm{~d} y}\right)^{2}\right) \rho_{1}=\left(\mathrm{e}^{-2 \mu_{0} \psi_{0}}\right) \psi_{1}-\left(\frac{\mathrm{d} \psi_{0}}{\mathrm{~d} y}\right)^{2} \frac{\partial \psi_{1}}{\partial y}$.

Integrating equation (A 6) and imposing the boundary condition (2.4) gives

$$
\psi_{0}(y)=\frac{1}{\mu_{0}} \ln \left(\cosh \left(\mu_{0} y\right)\right)-\frac{1}{2 \mu_{0}} \ln \left(\mu_{0}\right) .
$$

It follows that $\psi_{1}(x, y)$ and $\rho_{1}(x, y)$ must decay to zero as $y \rightarrow \infty$. At this stage, $\mu_{0}\left(M_{\infty}\right)$ remains undetermined. To proceed, (A 8), (A 7) and (A 5) are used to obtain a single equation for $\psi_{1}$. Boundary condition $(2.4)$, the change of variables $\zeta=\tanh \left(\mu_{0} y\right)$, and a cosine transform in $x$, where $s\left(\zeta ; \alpha_{s}\right)$ denotes the transform of $\psi_{1}(x, y)$, yields a singular Sturm-Liouville problem for $s\left(\zeta ; \alpha_{s}\right)$. Its eigenvalue is $\lambda=\left(\alpha_{s} / \mu_{0}\right)^{2}$, where $\alpha_{s}$ is the steady streamwise wavenumber. Using the equation for $s\left(\zeta ; \alpha_{s}\right)$ a singular Sturm-Liouville equation for the transform of the density perturbation, $r\left(\zeta ; \alpha_{s}\right)$, may be derived. Hence, $\mu_{0}$ is to be obtained from an eigensolution of the $O(\epsilon)$ equation for $\psi$ or $\rho$ :

$$
\begin{aligned}
& \frac{\mathrm{d}}{\mathrm{d} \zeta}\left(\frac{1-\zeta^{2}}{\zeta^{2}} \frac{\mathrm{d} r}{\mathrm{~d} \zeta}\right)-\lambda\left(\frac{1-M_{\infty}^{2} \zeta^{2}}{\zeta^{2}\left(1-\zeta^{2}\right)}\right) r=0, \\
& r^{\prime}\left(\zeta=0 ; \alpha_{s}, M_{\infty}\right)=r\left(\zeta=1 ; \alpha_{s}, M_{\infty}\right)=0 .
\end{aligned}
$$

Thus, $s\left(\zeta ; \alpha_{s}\right)$ and $r\left(\zeta ; \alpha_{s}\right)$ may be solved for and the inverse transforms taken to give

$$
\begin{gathered}
\lambda=1-M_{\infty}^{2}, \quad \alpha_{s}=1, \quad \mu_{0}=\frac{1}{\sqrt{1-M_{\infty}^{2}}} . \\
\psi_{1}(x, y)=\cos (x) \operatorname{sech}\left(\mu_{0} y\right)^{1-M_{\infty}^{2}}, \quad \rho_{1}(x, y)=\mu_{0} M_{\infty} \psi_{1}(x, y) .
\end{gathered}
$$

The parallel stream function, when $\epsilon \rightarrow 0$, is thus given by (A 8), with $\mu_{0}\left(M_{\infty}\right)$ given by (A 11). Hence, the limiting parallel shear flow for the CSV when $\epsilon \rightarrow 0$ with $M_{\infty}$ 
fixed may be obtained from its incompressible counterpart upon application of a Prandtl-Glauert stretching in the $y$-direction. Clearly, the solution branch terminates as $M_{\infty} \rightarrow 1$. The above expressions are expected to be uniformly valid in $M_{\infty}$, when $\epsilon \ll 1$, and agree with the numerical solution of MMP to six figures. Expanding $\mu_{0}\left(M_{\infty}\right)$ in (A 11) for $M_{\infty} \ll 1$ gives agreement with (2.8) to $O\left(M_{\infty}^{2}\right)$.

Equation (A9) may be used to link (A 12) to the neutrally stable density perturbation to a parallel compressible constant-density shear layer (just as Stuart (1967) linked his solution to the stable perturbation to a parallel hyperbolic incompressible shear layer). To see this, we first derive a single equation governing disturbances to the simple CD profile of $\S 4$, from which we can obtain neutral solutions for streamwise wavenumbers exceeding a critical value.

For the CD profile, (3.3)-(3.7) may be reduced to a single equations for $\rho^{\prime}(x, y, z, t)$ :

$$
\left(M_{\infty}^{2} \mathrm{~L}^{3}-\mathrm{L}\left(\mathrm{D}_{x}^{2}+\mathrm{D}_{y}^{2}+\mathrm{D}_{z}^{2}\right)+2 \frac{\mathrm{d} \bar{u}}{\mathrm{~d} y} \mathrm{D}_{x} \mathrm{D}_{y}\right) \rho^{\prime}(x, y, z, t)=0 .
$$

where in this Appendix

$$
\mathrm{L}=\frac{\partial}{\partial t}+\bar{u}(y) \frac{\partial}{\partial x}, \quad \mathrm{D}_{x}=\frac{\partial}{\partial x}, \quad \mathrm{D}_{y}=\frac{\partial}{\partial y}, \quad \mathrm{D}_{z}=\frac{\partial}{\partial z} .
$$

Setting the time derivatives in equation (A 13) to zero allows steady solutions to be obtained. For two-dimensional disturbances to a parallel flow, there is stability to any perturbation with streamwise wavenumber larger than the steady wavenumber; Lin (1953). Obtaining the steady solutions will allow steady (transitional) wavenumbers to be determined. Take the Fourier transform in $x$ and $z$, and denote the streamwise and spanwise wavenumbers at which the steady solutions may be found $\alpha_{s}$ and $\beta_{s}$. Define $\hat{\alpha}_{s}^{2}=\alpha_{s}^{2}+\beta_{s}^{2}, \hat{\alpha}_{s} \hat{M}_{\infty}=\alpha_{s} M_{\infty}$, and use the transformation $\zeta=\tanh \left(\omega_{h} y\right)$, to obtain the following singular Sturm-Liouville equation, with eigenvalue $\lambda=\hat{\alpha}_{s}^{2} / \omega_{h}^{2}$, for the steady transformed density perturbation, $R\left(\zeta ; \alpha_{s}, \beta_{s}\right)$ :

$$
\begin{gathered}
\frac{\mathrm{d}}{\mathrm{d} \zeta}\left(\frac{1-\zeta^{2}}{\zeta^{2}} \frac{\mathrm{d} R}{\mathrm{~d} \zeta}\right)-\lambda\left(\frac{1-\hat{M}_{\infty}^{2} \zeta^{2}}{\zeta^{2}\left(1-\zeta^{2}\right)}\right) R=0, \\
R(1)=R(-1)=0 .
\end{gathered}
$$

In the two-dimensional limit this equation is identical to (A9). For a non-trivial solution, the parameters $\left(\omega_{h}, \hat{\alpha}_{s}, \hat{M}_{\infty}\right)$ must be connected through the eigenvalue relationship, derived previously. This implies that

$$
\frac{1}{\omega_{h}^{2}}=\frac{\alpha_{s}^{2}\left(1-M_{\infty}^{2}\right)+\beta_{s}^{2}}{\left(\alpha_{s}^{2}+\beta_{s}^{2}\right)^{2}}
$$

When $\omega_{h}$ is specified, this condition defines a zero contour on which remaining parameters must lie. Thus, non-trivial steady disturbances to the hyperbolic tangent profile are obtained only for parameters $M_{\infty}, \alpha_{s}$ and $\beta_{s}$, which lie on the surface defined in $\left(\alpha, \beta, M_{\infty}\right)$ space. Disturbances which lie just inside the steady surface are amplified, while those just outside are neutral: Lin (1953) and Lessen et al. (1965). If the Mach number is also set to zero, the incompressible solution of Michalke (1965) is obtained.

Thus, at given $M_{\infty}$ the two-dimensional steady neutral stability point can be viewed as a bifurcation point from which the small-mass-flux CSV solution begins. Interpreting the steady stability point in this fashion provides an existence proof for 
steady three-dimensional supersonic solutions to the compressible Euler equations. Relation (A 17) indicates that steady supersonic perturbations do exist, but for nonzero values of $\beta_{s}$. Introducing $\beta_{s}$ in this equation allows $\alpha_{s}$ to remain real, or $\mu$ to remain finite, as the sonic threshold is crossed. If this point were to be viewed as a bifurcation point from which a three-dimensional steady vortex solution begins, then arclength continuation might be used to move into a regime where the full nonlinear Euler equations are solved.

\section{REFERENCES}

BAYLY, B. 1986 Three-dimensional instability of elliptical flow. Phys. Rev. Lett. 57, 2160-2163.

Bernal, L. \& Roshko, A. 1986 Streamwise vortex structure in plane mixing layers. J. Fluid Mech. 170, 499-525.

BiRCH, S. \& EGGERS, J. 1972 A critical review of the experimental data for developed free turbulent shear layers. NASA SP-321, pp. 11-40.

Blumen, W. 1970 Shear layer instabilities of an inviscid compressible fluid. J. Fluid Mech. 40, 769-781.

Blumen, W., Drazin, P. \& Billings, D. 1975 Shear layer instabilities of an inviscid compressible fluid. Part 2. J. Fluid Mech. 71, 305-316.

BogdanofF, D. 1983 Compressibility effects in turbulent shear layers. AIAA J. 21, 926-927.

BoYD, J. 1978a A Chebyshev polynomial method for computing analytic solutions to eigenvalue problems with applications to the anharmonic ocsillator. J. Math. Phys 19, 1445-1456.

BoYD, J. $1978 b$ Spectral and pseudospectral methods for eigenvalue and nonseperable boundary value problems. Mont. Weath. Rev. 106, 1192-1203.

BoYD, J. 1982 The optimization of convergence for Chebyshev polynomial methods in an unbounded domain. J. Comput. Phys. 45, 43-79.

Browand, F. \& Latigo, B. 1979 Growth of the two-dimensional mixing layers from a turbulent and nonturbulent boundary layer. Phys. Fluids 22, 1011-1019.

Brown, G. \& Roshko, A. 1974 On density effects and large structure in turbulent mixing layers. J. Fluid Mech. 64, 775-816.

Cain, A., Ferziger, J. \& Reynolds, W. 1984 Discrete orthogonal function expansions for non uniform grids using the fast Fourier transform. J. Comput. Phys. 56, 272-286.

Chandrsuda, C., Mehta, R., Weir, A. \& Bradshaw, P. 1978 Effect of free-stream turbulence on large structures in turbulent mixing layers. J. Fluid Mech. 85.

Chinzei, N., Masuya, G., Komuro, T., Murakami, A. \& Kudoi, K. 1986 Spreading of two-stream supersonic turbulent mixing layers. Phys. Fluids 29, 1345-1347.

Clemens, N. \& Mungal, M. 1995 Large-scale structure end entrainment in the supersonic mixing layer. J. Fluid Mech. 284, 171-216.

Goebel, S. \& Dutton, J. 1991 Experimental study of compressible turbulent mixing layers. AIAA J. 29, 538-546.

Hall, J., Dimotakis, P. \& Rosemann, H. 1993 Experiments in nonreacting compressible shear layers. AIAA J. 31, pp.

Kerswell, K. 2002 Elliptical Instability. Annu. Rev. Fluid Mech. 34, 83-113.

KlaAssen, G. \& Peltier, W. 1985 The onset of turbulence in finite amplitude kelvin-helmholtz billows. J. Fluid Mech. 155, 1-53.

Klatassen, G. \& Peltier, W. 1989 The role of transverse seconday instabilities in the evolution of free shear layers. J. Fluid Mech. 202, 367-402 (referred to herein as KP).

Klatssen, G. \& Peltier, W. 1991 The influence of stratification on secondary instability in free shear layers. J. Fluid Mech. 227, 71-106.

LAMB, H. 1932 Hydrodymanics, 6th edn. Cambridge Mathematical Library.

LANDMAN, M. \& SAFFMAN, P. 1987 The three-dimensional instability of strained vortices in a viscous fluid. Phys. Fluids 30, 2339-2342.

LEES, L. \& LIN, C. 1946 Investigation of the stability of the laminar boundary layer in a compressible fluid. Wash. Rep. 1115. Natl Adv. Comm. Aero. 
Lessen, M., Fox, J. \& ZIEN, H. 1965 On the inviscid stability of the laminar mixing of two parallel streams of compressible fluid. J. Fluid Mech. 23, 355-367.

Lessen, M., Fox, J. \& Zien, H. 1966 Stability of the laminar mixing of two parallel streams with respect to supersonic disturbances. J. Fluid Mech. 25, 737-742.

Lifschitz, A. \& Hameiri, E. 1991 Local stability conditions in fluid dunamics. Phys. Fluids A 3 , $2644-2651$.

LIN, C. 1953 On the stability of the laminar mixing region between two parallel streams in a gas. Wash. Rep. 2887. Natl Adv. Comm. Aero.

Lin, S. \& Corcos, G. 1984 The mixing layer: deterministic models of turbulent flow. Part 3. The effect of plain strain on the dynamics of streamwise vortices. J. Fluid Mech 141, 139-178.

Meiron, D., Moore, D. \& Pullin, D. 2000 On steady compressible flows with compact vorticity; the compressible Stuart vortex. J. Fluid Mech. 409, 29-49 (referred to herein as MMP).

Michalke, A. 1965 On spatially growing disturbances in an inviscid shear layer. J. Fluid Mech. 23, $521-544$.

Moore, D. \& Saffman, P. 1972 The density of organized vortices in a turbulent mixing layer. J. Fluid Mech. 64, 465-473.

Papamoschou, D. 1986 Experimental investigation of heterogeneous compressible shear layers. $\mathrm{PhD}$ thesis, California Institute of Technology.

Papamoschou, D. \& Bunyajitradulya, A. 1996 Evolution of large eddies in compressible shear layers. Phys. Fluids 9, 756-765.

Papamoschou, D. \& Roshko, A. 1986 The compressible turbulent shear layer: an experimental study. AIAA Paper 86-0162.

Papamoschou, D. \& Roshko, A. 1988 The compressible turbulent shear layer: an experimental study. J. Fluid Mech. 197, 453-477.

Pierrehumbert, R. 1986 Universal short-wave instability of two-dimensional eddies in an inviscid fluid. Phys. Rev. Lett. 57, 2157-2159.

Pierrehumbert, R. \& Widnall, S. 1982 The two- and three-dimensional instabilities of a spatially periodic shear layer. J. Fluid Mech. 114, 59-82 (referred to herein as PW).

RAGAB, S. \& WU, J. 1989 Linear instabilities in two-dimensionl compressible mixing layers. Phys. Fluids A 1, 957-966.

Rosenhead, L. 1930 The spread of vorticity in the wake behind a cylinder. Proc. R. Soc. Lond. A 127, 590-612.

Samimy, M. \& Elliott, G. 1990 Effects of compressibility in the characteristics of free shear layers. AIAA J. 28, 439-445.

SANDHAM, N. \& ReYNOLDS, W. 1990 Compressible mixing layer: linear theory and direct simulation. AIAA J. 28, 618-624.

SAndham, N. \& Reynolds, W. 1991 Three-dimensional simulations of large eddies in the compressible mixing layer. J. Fluid Mech. 224, 133-158.

Sirieix, M. \& Solignac, J. 1966 Contribution a l'etude experimentale de la couche de melange turbulent isobare d'un ecoulment supersonique. AGARD CP4.

SLEssor, M. 1998 Aspects of turbulent-shear-layer dynamics and mixing. PhD thesis, California Institute of Technology.

Slessor, M., Zhuang, M. \& Dimotakis, P. 2000 Turbulent shear-layer mixing: growth-rate compressibility scaling. J. Fluid Mech. 414, 35-45.

StUART, J. 1967 On finite amplitude oscillations in laminar mixing layers. J. Fluid Mech. 29, 417-440.

WALEFFE, F. 1990 On the three-dimensional instability of strained vortices. Phys. Fluids A 2, 76-80.

Winant, C. \& Browand, F. 1974 Vortex pairing: The mechanism of turbulent mixing layer growth at moderate reynolds number. J. Fluid Mech. 63, 237-255.

Zhuang, M. \& Dimotakis, P. 1995 Instability of wake dominated compressible mixing layers. Phys. Fluids 7, 2489-2495.

Zhuang, M., Dimotakis, P. \& Kubota, T. 1990a The effect of walls on spatially growing supersonic shear layers. Phys. Fluids A 2, 599-604.

Zhuang, M., Kubota, T. \& Dimotakis, P. $1990 \mathrm{~b}$ Instability of inviscid, compressible free shear layers. AIAA J. 28, 1728-1733. 